\title{
Nuclear three-body problem in the complex energy plane: Complex-scaling Slater method
}

\author{
A. T. Kruppa, ${ }^{1,2}$ G. Papadimitriou, ${ }^{3,1}$ W. Nazarewicz, ${ }^{1,4,5}$ and N. Michel ${ }^{6,1}$ \\ ${ }^{1}$ Department of Physics and Astronomy, University of Tennessee, Knoxville, Tennessee 37996, USA \\ ${ }^{2}$ Hungarian Academy of Sciences Institute for Nuclear Research, P.O. Box 51, H-4001 Debrecen, Hungary \\ ${ }^{3}$ Department of Physics, University of Arizona, Tucson, Arizona 85721, USA \\ ${ }^{4}$ Physics Division, Oak Ridge National Laboratory, Oak Ridge, Tennessee 37831, USA \\ ${ }^{5}$ Institute of Theoretical Physics, University of Warsaw, ul. Hoża 69, PL-00-681 Warsaw, Poland \\ ${ }^{6}$ National Superconducting Cyclotron Laboratory and Department of Physics and Astronomy, Michigan State University, \\ East Lansing, Michigan 48824, USA
}

(Received 28 October 2013; published 31 January 2014)

\begin{abstract}
Background: The physics of open quantum systems is an interdisciplinary area of research. The nuclear "openness" manifests itself through the presence of the many-body continuum representing various decay, scattering, and reaction channels. As the radioactive nuclear beam experimentation extends the known nuclear landscape toward the particle drip lines, the coupling to the continuum space becomes exceedingly more important. Of particular interest are weakly bound and unbound nuclear states appearing around particle thresholds. Theories of such nuclei must take into account their open quantum nature.

Purpose: To describe open quantum systems, we introduce a complex-scaling (CS) approach in the Slater basis. We benchmark it with the complex-energy Gamow shell model (GSM) by studying energies and wave functions of the bound and unbound states of the two-neutron halo nucleus ${ }^{6} \mathrm{He}$ viewed as an $\alpha+n+n$ cluster system.

Methods: Both CS and GSM approaches are applied to a translationally invariant Hamiltonian with the two-body interaction approximated by the finite-range central Minnesota force. In the CS approach, we use the Slater basis, which exhibits the correct asymptotic behavior at large distances. To extract particle densities from the back-rotated CS solutions, we apply the Tikhonov regularization procedure, which minimizes the ultraviolet numerical noise.

Results: We show that the CS-Slater method is both accurate and efficient. Its equivalence to the GSM approach has been demonstrated numerically for both energies and wave functions of ${ }^{6} \mathrm{He}$. One important technical aspect of our calculation was to fully retrieve the correct asymptotic behavior of a resonance state from the complex-scaled (square-integrable) wave function. While standard applications of the inverse complex transformation to the complex-rotated solution provide unstable results, the stabilization method fully reproduces the GSM benchmark. We also propose a method to determine the smoothing parameter of the Tikhonov regularization.

Conclusions: The combined suite of CS-Slater and GSM techniques has many attractive features when applied to nuclear problems involving weakly bound and unbound states. While both methods can describe energies, total widths, and wave functions of nuclear states, the CS-Slater method-if it can be applied — can provide additional information about partial energy widths associated with individual thresholds.
\end{abstract}

DOI: 10.1103/PhysRevC.89.014330

\section{INTRODUCTION}

The physics of open quantum systems spans many areas of research, ranging from optical physics to nanoscience, to atomic and nuclear physics. Of particular interest are long-lived metastable states and broad resonances: they carry rich information about localized nucleonic states confined to the nuclear interior, about the multichannel environment of scattering and decaying states, and about the coupling between these two spaces. With exciting advances in radioactive beam experimentation worldwide, many weakly bound isotopes inhabiting the outskirts of the nuclear landscape can now be reached; they provide a fertile territory for studying generic properties of open quantum systems [1].

To develop a microscopic theoretical framework that would unify structural and reaction-theoretical aspects of the nuclear many-body system remains a challenge. A step in this direction is the unification of bound states and resonant phenomena, often enabled by high-performance computing, and there has been excellent progress in this area [2-9].
PACS number(s): 21.60.Gx, 21.60.Cs, 21.10.Gv, 02.60.-x

One possible strategy in this area is to relate the resonance parameters directly to the complex-energy eigenvalues of the effective Hamiltonian. To this end, one can solve the many-body eigenproblem with the Hermitian Hamiltonian by imposing specific boundary conditions [10], or one can construct a manifestly non-Hermitian effective Hamiltonian [11-13]. In both cases, the eigenstates that appear below the particle threshold are bound, and the complex-energy states above the threshold represent the many-body continuum.

The Gamow shell model (GSM) [10] and complex-scaling (CS) [14-16] methods deal with effective non-Hermitian Hamiltonians. In the GSM, one starts with a Hermitian Hamiltonian and by imposing outgoing boundary conditions one ends up with a complex-symmetric Hamiltonian matrix. In the CS method, a non-Hermitian Hamiltonian appears as a result of a complex rotation of coordinates. The corresponding nonunitary transformation is characterized by a real parameter $\vartheta$. The transformed eigenstates are square integrable; this is a very attractive feature from a computational point of view. Unfortunately, since the eigenvectors depend on $\vartheta$, they 
cannot be directly compared with the eigenfunctions of the original Hamiltonian. To obtain the wave functions from the CS solutions, the so-called back-rotation must be employed. Since in most cases the eigenproblem is solved numerically, the back-rotation constitutes an ill-posed inverse problem and high-frequency ultraviolet noise appears $[17,18]$. We are aware of at least two attempts [19,20] to overcome this problem. When the original wave function is reconstructed by means of the Padé approximation [19], several calculations with different $\vartheta$ values can be carried out to perform the analytical continuation. In Ref. [20], special properties of the applied basis set were utilized to cure the errors of the back-rotated wave function. (Reproducing the correct behavior of the wave function is of particular importance in the context of particle densities, which carry information about many-body correlations and also about fundamental properties of unbound states.) In this work, we will present a new approach to the problem of back-rotation. Our procedure does not depend on the type of basis set used, and it is based on sound mathematical foundations.

The CS method has been successfully applied in quantum chemistry to solve many-body problems with an extremely high accuracy [14,15,21-23] and also in nuclear physics and in calculations of resonance parameters [24,25] and cluster systems [16,26-28]. In the nuclear three-body calculations, mainly Jacobi coordinates have been employed. In the cluster orbital shell model [16], besides the "V"-type coordinate, also a "T"-type Jacobi coordinate has been used in order to incorporate correlations. In the field of quantum chemistry, on the other hand, mainly Hylleraas-type functions [29,30] are used, and the achieved accuracy for the helium atom is spectacular [31-33].

In our CS calculations, we employ the Slater basis set [34]. The Slater wave functions have a correct asymptotic behavior, making them a perfect choice for the description of weakly bound systems. A basis set of similar type, the Coulomb-Sturmian functions, has been recently introduced into the no-core shell model framework [35] and has been employed in reaction calculations [36]. Those functions are in fact linear superpositions of Slater orbits.

In this work, the precision of the new CS-Slater method is tested against the results of the GSM calculations. For the sake of benchmarking, we consider the energies and wave functions of the $0_{1}^{+}$and $2_{1}^{+}$states of ${ }^{6} \mathrm{He}$. The paper is organized as follows. Section II describes the Hamiltonian used, manybody methods, and configuration spaces employed. In Sec. III we discuss the difficulties related to the back-rotation of the CS wave function and introduce the necessary regularization scheme. Section IV presents the results for ${ }^{6} \mathrm{He}$ and the details of the CS-GSM benchmarking. Finally, conclusions and future plans are contained in Sec. V.

\section{MODELS AND METHODS}

\section{A. Three-body Hamiltonian}

For the description of the ground and excited state of ${ }^{6} \mathrm{He}$ we assume a cluster $(\alpha+n+n)$ picture of the nucleus. Consequently, we consider a system of three particles with masses $m_{i}$ and single-particle coordinates $\boldsymbol{r}_{i}$, where $i=1,2$ for neutrons and $i=3$ for the $\alpha$ core. We introduce the relative coordinates $\boldsymbol{r}_{i j}=\boldsymbol{r}_{i}-\boldsymbol{r}_{j}$ and $r_{i j}=\left|\boldsymbol{r}_{i j}\right|$. The system Hamiltonian in the center-of-mass frame reads

$$
\begin{aligned}
H= & -\frac{\hbar^{2}}{2 \mu_{1}} \triangle_{\boldsymbol{r}_{13}}-\frac{\hbar^{2}}{2 \mu_{2}} \triangle_{\boldsymbol{r}_{23}}-\frac{\hbar^{2}}{m_{3}} \nabla_{\boldsymbol{r}_{13}} \nabla_{\boldsymbol{r}_{23}} \\
& +V_{12}\left(\boldsymbol{r}_{12}\right)+V_{13}\left(\boldsymbol{r}_{13}\right)+V_{23}\left(\boldsymbol{r}_{23}\right),
\end{aligned}
$$

where the reduced masses are

$$
\mu_{1}=\frac{m_{1} m_{3}}{m_{1}+m_{3}}, \quad \mu_{2}=\frac{m_{2} m_{3}}{m_{2}+m_{3}} .
$$

It is worth noting that the Hamiltonian (1) represents the intrinsic properties of the system; i.e., it is free from the spurious center-of-mass motion. After introduction of the single-neutron Hamiltonian,

$$
H_{i 3}(\boldsymbol{r})=-\frac{\hbar^{2}}{2 \mu_{i}} \triangle_{\boldsymbol{r}}+V_{i 3}(\boldsymbol{r}) \quad(i=1,2),
$$

the Hamiltonian (1) can be written as

$$
H=H_{13}\left(\boldsymbol{r}_{13}\right)+H_{23}\left(\boldsymbol{r}_{23}\right)+V_{12}\left(\boldsymbol{r}_{12}\right)-\frac{\hbar^{2}}{m_{3}} \nabla_{\boldsymbol{r}_{13}} \nabla_{\boldsymbol{r}_{23}},
$$

where the last term represents a two-body recoil term, which originates from the transformation to the relative coordinate frame.

\section{B. Complex-scaling method}

The key element of the CS method is the complexscaling operator $U(\vartheta)$, which transforms an arbitrary function $\chi\left(\boldsymbol{r}_{13}, \boldsymbol{r}_{23}\right)$ according to

$$
U(\vartheta) \chi\left(\boldsymbol{r}_{13}, \boldsymbol{r}_{23}\right)=e^{i 3 \vartheta} \chi\left(e^{i \vartheta} \boldsymbol{r}_{13}, e^{i \vartheta} \boldsymbol{r}_{23}\right) .
$$

The transformed Shrödinger equation becomes

$$
H_{\vartheta} \Psi_{\vartheta}=E \Psi_{\vartheta}
$$

where

$$
H_{\vartheta}=U(\vartheta) H U(\vartheta)^{-1}
$$

is a complex-scaled Hamiltonian

$$
\begin{aligned}
H_{\vartheta}= & e^{-2 i \vartheta}\left(-\frac{\hbar^{2}}{2 \mu_{1}} \triangle_{\boldsymbol{r}_{13}}-\frac{\hbar^{2}}{2 \mu_{2}} \Delta_{\boldsymbol{r}_{23}}-\frac{\hbar^{2}}{m_{3}} \nabla_{\boldsymbol{r}_{13}} \nabla_{\boldsymbol{r}_{23}}\right) \\
& +V_{12}\left(e^{i \vartheta} \boldsymbol{r}_{12}\right)+V_{13}\left(e^{i \vartheta} \boldsymbol{r}_{13}\right)+V_{23}\left(e^{i \vartheta} \boldsymbol{r}_{23}\right) .
\end{aligned}
$$

The eigenfunctions $\Psi\left(\boldsymbol{r}_{13}, \boldsymbol{r}_{23}\right)$ and $\Psi_{\vartheta}\left(\boldsymbol{r}_{13}, \boldsymbol{r}_{23}\right)$ of the Hamiltonians (1) and (8) satisfy the following relation:

$$
\Psi_{\vartheta}\left(\boldsymbol{r}_{13}, \boldsymbol{r}_{23}\right)=e^{i 3 \vartheta} \Psi\left(e^{i \vartheta} \boldsymbol{r}_{13}, e^{i \vartheta} \boldsymbol{r}_{23}\right)
$$

or the so-called back-rotation relation

$$
\Psi\left(\boldsymbol{r}_{13}, \boldsymbol{r}_{23}\right)=e^{-i 3 \vartheta} \Psi_{\vartheta}\left(e^{-i \vartheta} \boldsymbol{r}_{13}, e^{-i \vartheta} \boldsymbol{r}_{23}\right) .
$$

According to the Aguilar-Balslev-Combes theorem [37,38], the resonant solutions of Eq. (6) are square integrable. This feature makes it possible to use bound-state methods to solve (6), including configuration interaction [14,15], Faddeev and Faddeev-Yakubovsky [39,40], and the coupled cluster method [41]. Because the resonant states are square integrable, they can be normalized to one. As illustrated in Fig. 1, the spectrum of the rotated Hamiltonian (7) consists of bound and unbound 


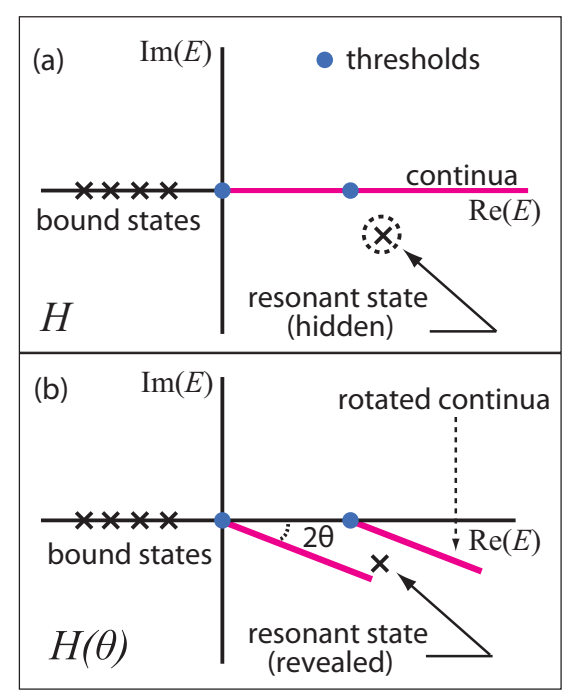

FIG. 1. (Color online) Illustration of the complex-scaling transformation of a many-body Hamiltonian. Bound states and many-body thresholds are invariant. Resonant eigenvalues corresponding to poles of the resolvent or the $S$ matrix are "hidden" on a sheet with $\vartheta=0$ (a) but are exposed if the cuts associated with many-body continua are rotated (b) [42].

states. The continuum part of the spectrum is represented by cuts in the complex-energy plane at an angle $2 \vartheta$ with the real-energy axis, originating at many-body thresholds. The resonant spectrum consists of bound states lying on the negative real energy axis and positive-energy resonances. One attractive feature of the CS method is that one does not need to apply directly any boundary condition to obtain the resonant states. Through the CS transformation $U(\vartheta)$, all resonant wave functions have decaying asymptotic behavior. Even though the solution of the complex-rotated Hamiltonian $H_{\vartheta}$ is square integrable, the back-rotated wave function is an outgoing solution of the Schrödinger equation with the original Hamiltonian $H$. The back-rotation transformation, or analytical continuation, will be investigated in the following.

While the rotated nonresonant continuum states depend on the rotation angle, resonant states should be independent of $\vartheta$. In practical applications, however, Eq. (6) cannot be solved exactly and usually a truncated basis set is adopted. As a consequence, the positions of resonant states move slightly with $\vartheta$ and/or the size of the (truncated) basis. Since the dependence on $\vartheta$ is radically different for the continuum spectrum and the resonant states, there exist practical techniques to identify the resonance solutions. One of them is the so-called $\vartheta$-trajectory method: using the generalization of the virial theorem to complex energies, one finds that the resonant solution must change little with $\vartheta$ around a certain value of $\vartheta=\vartheta_{\text {opt }}$. In this work, we checked carefully the dependence of resonant states on both $\vartheta$ and basis parameters.

\section{Slater-basis expansion}

To solve the CS problem, we use a finite Slater-type basis set [34]. Namely, the eigenstate of the original Hamiltonian is assumed to be

$$
\Psi^{J M}\left(\boldsymbol{x}_{13}, \boldsymbol{x}_{23}\right)=\sum_{\{l j\}} \sum_{A} C_{A}^{\{l j\}} \chi_{A}^{\{l j\}}\left(r_{13}, r_{23}\right) \mathcal{Y}_{\{l j\}}^{J M T T_{z}}\left(\boldsymbol{x}_{13}, \boldsymbol{x}_{23}\right),
$$

where the linear expansion coefficients $C_{A}^{\{l j\}}$ are determined by the Rayleigh-Ritz variational principle. Here $\boldsymbol{x}_{13}$ and $\boldsymbol{x}_{23}$ denote the spatial and spin-isospin coordinates of first and second particle, respectively. For brevity we introduce the compact notation $\{l j\}=l_{13}, j_{13}, l_{23}, j_{23}$. Furthermore, we introduce the spin-isospin part

$$
\begin{aligned}
\mathcal{Y}_{\{l j\}}^{J M T T_{z}}\left(\boldsymbol{x}_{13}, \boldsymbol{x}_{23}\right)= & \chi_{T T_{z}}(1,2)\left[\left[\mathcal{Y}_{l_{13}}\left(\boldsymbol{r}_{13}\right) \otimes \chi_{1 / 2}(1)\right]^{j_{13}}\right. \\
& \left.\otimes\left[\mathcal{Y}_{l_{23}}\left(\boldsymbol{r}_{23}\right) \otimes \chi_{1 / 2}(2)\right]^{j_{23}}\right]^{J M},
\end{aligned}
$$

where the solid spherical harmonics are $\mathcal{Y}_{l m}(\boldsymbol{r})=r^{l} Y_{l m}(\hat{\boldsymbol{r}})$. The symbol $[\otimes]^{J M}$ denotes the angular momentum coupling and $\hat{\boldsymbol{r}}_{i j}$ stands for the angular coordinates of $\boldsymbol{r}_{i j}$. The total isospin and single-nucleon spin functions are, respectively, denoted by $\chi_{T, T_{z}}(1,2)$ and $\chi_{1 / 2}(i) i=1,2$.

For the radial part of the wave function we use the product of Slater-type functions:

$$
\chi_{A}^{\{l j\}}\left(r_{13}, r_{23}\right)=r_{13}^{n} e^{-\alpha r_{13}} r_{23}^{m} e^{-\beta r_{23}},
$$

where the nonlinear parameters of the basis may depend on the quantum numbers $\{l j\}$ and they are denoted by $A=$ $\{\alpha, n, \beta, m\}$. At this point, we neglect the internucleon distance $r_{12}$ in the radial part in order to span the same subspace of the Hilbert space as the GSM. (When the three-body wave function does not depend on the interparticle distance $r_{12}$ one refers to the resulting set as the Slater basis. If all three coordinates are considered, the basis set is called a Hylleraas basis.)

In the LS coupling, the wave function (11) can be written in the form

$$
\begin{aligned}
\Psi^{J M}\left(\boldsymbol{x}_{13}, \boldsymbol{x}_{23}\right)= & \sum_{\{l j\}} \sum_{L S} \sum_{A} C_{A}^{\{l j\}} \chi_{A}^{\{l j\}}\left(r_{13}, r_{23}\right) \\
& \times \gamma_{L S}(\{l j\})\left[\mathcal{Y}_{l_{13} l_{23}}^{L}\left(\boldsymbol{r}_{13}, \boldsymbol{r}_{23}\right) \otimes \chi_{S}(1,2)\right]^{J M} \\
& \times \chi_{T T_{2}}(1,2),
\end{aligned}
$$

where

$$
\mathcal{Y}_{l_{1} l_{2}}^{L M}\left(\boldsymbol{r}_{1}, \boldsymbol{r}_{2}\right)=\sum_{m_{1}, m_{2}}\left\langle l_{1} m_{1}, l_{2} m_{2} \mid L M\right\rangle \mathcal{Y}_{l_{1}, m_{1}}\left(\boldsymbol{r}_{1}\right) \mathcal{Y}_{l_{2}, m_{2}}\left(\boldsymbol{r}_{2}\right)
$$

are the bipolar harmonics, $\chi_{S S_{z}}(1,2)$ are coupled total spin functions, and $\gamma_{L S}(\{l j\})$ are recoupling coefficients [43]. In the case of a many-body system, the trial wave function is expanded in a many-body antisymmetric basis in a coupled or uncoupled scheme. In our formalism, we use the fully antisymmetrized wave functions expressed in both LS- and JJ-coupling schemes. The trial wave function of the CS Hamiltonian has the same form as Eq. (11):

$$
\begin{aligned}
& \Psi_{\vartheta}^{J M}\left(\boldsymbol{x}_{13}, \boldsymbol{x}_{23}\right) \\
& =\sum_{\{l j\}} \sum_{A} C_{A}^{\{l j\}}(\vartheta) \chi_{A}^{\{l j\}}\left(r_{13}, r_{23}\right) \mathcal{Y}_{\{l j\}}^{J M T T_{z}}\left(\boldsymbol{x}_{13}, \boldsymbol{x}_{23}\right),
\end{aligned}
$$


but the expansion coefficients $C_{A}^{\{l j\}}(\vartheta)$ now depend on $\vartheta$ and they are determined by using the generalized variational principle.

\section{Two-body matrix elements in CS}

Since the CS wave function is of Slater type, one needs to develop a technique to compute two-body matrix elements (TBMEs). In the following, we briefly review a method developed in the context of atomic physics applications [44-46].

Since we employ the LS coupling scheme, for TBMEs we need to consider integrals of the type

$$
\begin{aligned}
\left\langle A^{\prime}\left\{l^{\prime} j^{\prime}\right\}\left|V_{12}\right| A\{l j\}\right\rangle= & \int d \tau \chi_{A^{\prime}}^{\left\{l^{\prime} j^{\prime}\right\}}\left(r_{13}, r_{23}\right) \mathcal{Y}_{l_{13}^{\prime} l_{23}^{\prime}}^{L}\left(\hat{\boldsymbol{r}}_{13}, \hat{\boldsymbol{r}}_{23}\right)^{*} \\
& \times V_{12}\left(r_{12}\right) \chi_{A}^{\{l j\}}\left(r_{13}, r_{23}\right) \mathcal{Y}_{l_{13} l_{23}}^{L}\left(\hat{\boldsymbol{r}}_{13}, \hat{\boldsymbol{r}}_{23}\right) .
\end{aligned}
$$

To compute (16), we make a coordinate transformation to the three scalar relative coordinates $r_{12}, r_{13}, r_{23}$ and three Euler angles $(\Omega)$ corresponding to a triangle formed by three particles. The volume element $d \tau=d \boldsymbol{r}_{13} d \boldsymbol{r}_{13}$ can be then written as $d \tau_{r} d \Omega$, where the radial volume element is given by $d \tau_{r}=d r_{12} d r_{13} d r_{23} r_{12} r_{13} r_{23}$, and $d \Omega$ corresponds to the angular volume element involving the Euler angles. The angular integral

$$
\begin{aligned}
& W_{l_{1}^{\prime} l_{2}^{\prime}, l_{1} l_{2}}^{L}\left(r_{12}, r_{13}, r_{23}\right) \\
& \quad=\int d \Omega \mathcal{Y}_{l_{1}^{\prime} l_{2}}^{L}\left(\boldsymbol{r}_{13}, \boldsymbol{r}_{23}\right)^{*} \mathcal{Y}_{l_{1} l_{2}}^{L}\left(\boldsymbol{r}_{13}, \boldsymbol{r}_{23}\right)
\end{aligned}
$$

can be calculated analytically [46], and the result is

$$
\begin{aligned}
& W_{l_{1}^{\prime}, l_{2}^{\prime}, l_{1}, l_{2}}^{L}\left(r_{12}, r_{13}, r_{23}\right) \\
& \quad=r_{13}^{l_{1}+l_{1}^{\prime}} r_{23}^{l_{2}+l_{2}^{\prime}} \sum_{\lambda} A\left(l_{1}^{\prime}, l_{2}^{\prime}, l_{1}, l_{2}, L, \lambda\right) P_{\lambda}\left(\frac{r_{13}^{2}+r_{23}^{2}-r_{12}^{2}}{2 r_{13} r_{23}}\right),
\end{aligned}
$$

where

$$
\begin{aligned}
A\left(l_{1}^{\prime}, l_{2}^{\prime}, l_{1}, l_{2}, L, \lambda\right) \\
=\frac{1}{2}(-1)^{L} \hat{l_{1}} \hat{l_{2}} \hat{l}_{1}^{\prime} \hat{l_{2}^{\prime}}(-1)^{\lambda}(2 \lambda+1)\left(\begin{array}{ccc}
l_{1}^{\prime} & l_{1} & \lambda \\
0 & 0 & 0
\end{array}\right) \\
\quad \times\left(\begin{array}{ccc}
l_{2}^{\prime} & l_{2} & \lambda \\
0 & 0 & 0
\end{array}\right)\left\{\begin{array}{lll}
l_{1} & l_{2} & L \\
l_{2}^{\prime} & l_{1}^{\prime} & \lambda
\end{array}\right\},
\end{aligned}
$$

with $\hat{j} \equiv \sqrt{2 j+1}$. The presence of the Legendre polynomial $P_{\lambda}$ in Eq. (18) shows that the function $W_{l_{1}, l_{2}, l_{1}^{\prime}, l_{2}^{\prime}}^{L}\left(r_{12}, r_{13}, r_{23}\right)$ is a multinomial in the variables $r_{12}, r_{13}$, and $r_{23}$. The interaction matrix element (16) can now be written in the compact form

$$
\begin{aligned}
& \left\langle A^{\prime}\left\{l^{\prime} j^{\prime}\right\}\left|V_{12}\right| A\{l j\}\right\rangle \\
& =\int_{0}^{\infty} d r_{13} r_{13} \int_{0}^{\infty} d r_{23} r_{23} \int_{\left|r_{13}-r_{23}\right|}^{r_{13}+r_{23}} d r_{12} r_{12} \\
& \quad \times \chi_{A^{\prime}}^{\left\{l^{\prime} j^{\prime}\right\}}\left(r_{13}, r_{23}\right) \chi_{A}^{\{l j\}}\left(r_{13}, r_{23}\right) \\
& \quad \times V_{12}\left(r_{12}\right) W_{l_{13}^{\prime}, l_{23}^{\prime}, l_{13}, l_{23}\left(r_{12}, r_{13}, r_{23}\right) .}
\end{aligned}
$$

Finally, we determine the radial integrals. By using the functional form of the basis (12) and the dependence of the function $W_{l_{1}, l_{2}, l_{1}^{\prime}, l_{2}^{\prime}}^{L}\left(r_{12}, r_{13}, r_{23}\right)$ on the integration variables, it follows that the building block of the calculation is the integral

$$
\begin{aligned}
& I^{(\lambda)}\left(n_{13}, n_{23}\right) \\
& =\int_{0}^{\infty} d r_{13} \int_{0}^{\infty} d r_{23} \int_{\left|r_{13}-r_{23}\right|}^{r_{13}+r_{23}} d r_{12} r_{12} r_{13}^{n_{13}} r_{23}^{n_{23}} \\
& \quad \times V_{12}\left(r_{12}\right) P_{\lambda}\left(\frac{r_{13}^{2}+r_{23}^{2}-r_{12}^{2}}{2 r_{13} r_{23}}\right) \exp \left(-a_{13} r_{13}-a_{23} r_{23}\right),
\end{aligned}
$$

where

$$
a_{13}=\alpha^{\prime}+\alpha, \quad a_{23}=\beta^{\prime}+\beta,
$$

and

$$
n_{13}=n^{\prime}+l_{13}^{\prime}+n+l_{13}+1, n_{23}=m^{\prime}+l_{23}^{\prime}+m+l_{23}+1 \text {. }
$$

The integral (21) can be easily calculated if the form factor of the interaction is exponential, Yukawa-like, or Coulomb [47]. For a Gaussian form factor (e.g., Minnesota force), the integral (21) is more involved and the relevant expressions are given in the Appendix.

\section{Gamow shell model}

The Gamow shell model is a complex-energy configuration interaction method [10], where the many-body Hamiltonian is diagonalized in a one-body Berggren ensemble [48] that contains both resonant and nonresonant states. The total GSM wave function is expanded in a set of basis states similar to Eq. (11). The basis functions $\psi_{l j}^{(\alpha)}(r)$ can here be represented by the eigenfunctions of a single-particle (s.p.) Hamiltonian (3) with a finite-depth potential $V(r)$ :

$$
\begin{aligned}
& \left(-\frac{\hbar^{2}}{2 \mu} \triangle_{r}+V(r)\right) \psi_{l j}^{(\alpha)}(r)\left[Y_{l}(\hat{\boldsymbol{r}}) \otimes \chi_{1 / 2}(1)\right]^{j m} \\
& =\epsilon_{\alpha} \psi_{l j}^{(\alpha)}(r)\left[Y_{l}(\hat{\boldsymbol{r}}) \otimes \chi_{1 / 2}(1)\right]^{j m} .
\end{aligned}
$$

The resonant eigenstates (bound states and resonances), which correspond to the poles of the scattering $S$ matrix, are obtained by a numerical integration of the radial part of Eq. (24) by assuming the outgoing boundary conditions

$$
\psi(r) \stackrel{r \rightarrow 0}{=} r^{l+1}, \quad \psi(r) \stackrel{r \rightarrow \infty}{=} H_{l}^{+}(k r),
$$

where $H_{l}(k r)$ is a Hankel function (or Coulomb function for protons). The resulting s.p. energies $\epsilon_{\alpha}$ and the associated linear momenta $\left(k_{\alpha}=\sqrt{2 m e_{\alpha}} / \hbar\right)$ are in general complex. As illustrated in Fig. 2, bound states are located on the imaginary momentum axis in the complex $k$ plane whereas the resonances are located in its fourth quadrant. The s.p. Hamiltonian also generates nonresonant states, which are solutions obeying scattering boundary conditions. The resonant and nonresonant states form a complete set (Berggren ensemble) $[48,49]$ :

$$
\sum_{b, r}\left|\psi_{b, r}^{\alpha}\right\rangle\left\langle\psi_{b, r}^{\alpha}\left|+\int_{L_{+}} d k\right| \psi_{k}^{\alpha}\right\rangle\left\langle\psi_{k}^{\alpha}\right|=1,
$$




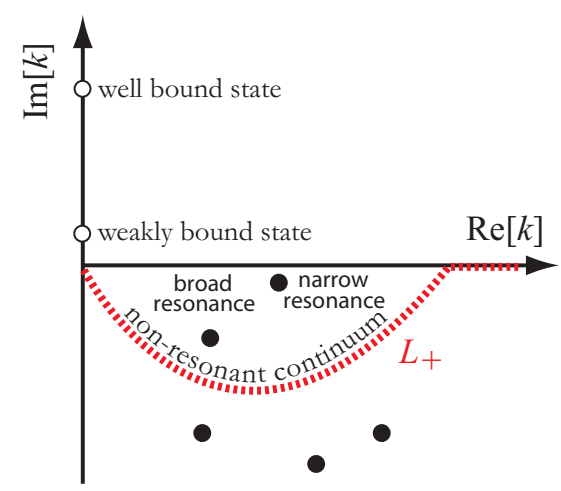

FIG. 2. (Color online) Berggren ensemble in the complex $k$ plane used to generate the s.p. basis of the GSM.

which is a s.p. basis of the GSM. In Eq. (26) $b$ (=bound) and $r$ (=resonance) are the resonant states, and the nonresonant states are distributed along a complex contour $L_{+}$. In our implementations, the continuum integral is discretized by using a Gauss-Legendre quadrature. The shape of the contour is arbitrary. The practical condition is that the contour should enclose narrow resonances for a particular partial wave. Additionally, the contour is extended up to a certain momentum cutoff $k_{\max }$. Then convergence of results is checked with respect to both the number of shells and the s.p. cutoff. For a sufficient number of points (shells), the basis (26) satisfies the completeness relation to a very high accuracy.

The total wave function is expanded in the complete set of the Berggren's ensemble:

$$
\begin{aligned}
& \Psi^{J M}\left(\boldsymbol{x}_{13}, \boldsymbol{x}_{23}\right) \\
& =\sum_{\{l j\}} \sum_{n} \sum_{m} C_{\{l j\}}^{(n, m)} \psi_{l_{13} j_{13}}^{(n)}\left(r_{13}\right) \psi_{l_{23} j_{23}}^{(m)}\left(r_{23}\right) \mathcal{Y}_{\{l j\}}^{J M T T_{z}}\left(\boldsymbol{x}_{13}, \boldsymbol{x}_{23}\right) .
\end{aligned}
$$

The resonant GSM wave function is normalized to one by using the external complex-scaling technique [10]. Comparing Eqs. (27) and (11), we notice that the GSM and CS-Slater wave functions differ by their radial parts. The expansion coefficients $C_{l j}^{(n)}$,s are determined variationally from the eigenvalue problem

$$
\sum_{\alpha_{1}^{\prime} \alpha_{2}^{\prime}}\left(H_{\alpha_{1} \alpha_{2} \alpha_{1}^{\prime} \alpha_{2}^{\prime}}-E C_{\alpha_{1}^{\prime} \alpha_{2}^{\prime}}\right)=0
$$

where $\alpha$ indices represent the s.p. $n l j$ quantum numbers. Since the basis is in general complex, $H_{\alpha_{1} \alpha_{2} \alpha_{1}^{\prime} \alpha_{2}^{\prime}}$ is a nonHermitian complex symmetric matrix. The Berggren ensemble involves functions which are not $L^{2}$ integrable. Consequently, normalization integrals and matrix elements of operators are calculated via the external complex-scaling technique [50].

The GSM Hamiltonian is given by Eq. (4). The s.p. potential $V(r)=V_{13}(r)=V_{23}(r)$ represents the interaction between the $\alpha$ core and the neutron, and $\mu=\mu_{1}=\mu_{2}$. The same interaction $V(r)$ is also used to generate the s.p. basis (24).

\section{Two-body matrix elements in the GSM}

Once the basis is generated one needs to calculate TBMEs in the Berggren basis. Since the Berggren basis is obtained numerically, the standard Brody-Moshinsky bracket technology [51], developed in the context of the harmonic oscillator (HO) s.p. basis, cannot be employed. To overcome this difficulty, we expand the nucleon-nucleon (NN) interaction in a truncated HO basis [52]:

$$
V_{N N}=\sum_{\alpha \beta \gamma \delta}^{N_{\max }}|\alpha \beta\rangle\left\langle\alpha \beta\left|V_{N N}\right| \gamma \delta\right\rangle\langle\gamma \delta| .
$$

The TBMEs in the Berggren ensemble are given by

$$
\left\langle\tilde{a b}\left|V_{N N}\right| c d\right\rangle=\sum_{\alpha \beta \gamma \delta}^{n_{\max }}\langle\tilde{a} b \mid \alpha \beta\rangle\left\langle\alpha \beta\left|V_{N N}\right| \gamma \delta\right\rangle\langle\gamma \delta \mid c d\rangle,
$$

where the Latin letters denote Berggren s.p. wave functions and Greek letters denote HO states. Due to the Gaussian fall-off of HO states, no external complex scaling is needed for the calculation of the overlaps $\langle\alpha \beta \mid a b\rangle$. Moreover, matrix elements $\left\langle\alpha \beta\left|V_{N N}\right| \gamma \delta\right\rangle$ of the NN interaction in the HO basis can be conveniently calculated by using the Brody-Moshinsky technique [51]. This method of treating the TBMEs of the interaction is similar to the technique based on a separable expansion of the potential [53]. The HO basis depends on the oscillator length $b$, which is an additional parameter. However, as was demonstrated in Refs. [52,54], GSM eigenvalues and eigenfunctions converge for a sufficient number of $n_{\max }$, and the dependence of the results on $b$ is negligible. We shall return to this point in Sec. IV A below.

\section{Model space of the GSM}

The CS and GSM calculations for the $0^{+}$ground state (g.s.) of ${ }^{6} \mathrm{He}$ have been performed in a model space of four partial waves: $p_{3 / 2}, p_{1 / 2}, s_{1 / 2}$, and $d_{5 / 2}$. The Berggren basis consists of the $0 p_{3 / 2}$ resonant state, which is found at an energy of $0.737-i 0.292 \mathrm{MeV}$ and the $p_{3 / 2}$ complex contour in order to satisfy the Berggren's completeness relation. In the actual calculation, we used a triangular contour located in the fourth quadrant of the complex $k$ plane that includes the $0 p_{3 / 2}$ singleparticle resonance. The momentum cutoff used was $k_{\max }=$ $3.5 \mathrm{fm}^{-1}$.

The remaining partial waves $p_{1 / 2}, s_{1 / 2}$, and $d_{5 / 2}$ are taken along the real axis. Each contour is discretized with 60 points; hence, our one-body space consists of a total of 241 neutron shells. Within such a basis, results are independent of the contour extension in $k$ space. The finite-range Minnesota interaction was expanded in a set of $\mathrm{HO}$ states. For the g.s., when a relatively large set of HO quanta is used, the dependence of the results on the HO parameter $b$ is negligible. We took $b=2 \mathrm{fm}$ and we used all HO states with up to $n_{\max }=18$ radial nodes. Since the $s$ wave enters the Berggren ensemble, in order to satisfy the Pauli principle between core and valence particles we project out the Pauli forbidden $0 s_{1 / 2}$ state $(b=1.4 \mathrm{fm})$ using the Saito orthogonality condition model [55].

For the excited unbound $2^{+}$state of ${ }^{6} \mathrm{He}$ we limit ourselves to a $p_{3 / 2}$ model space. As concluded in Ref. [56], the structure 
of this state is dominated by a $\left(p_{3 / 2}\right)^{2}$ parentage. Moreover, in this truncated space the neutron radial density becomes less localized since the $2^{+}$state becomes less bound when the model space is increased. The width of this state increases from $\sim 250 \mathrm{keV}$ in the $\left(p_{3 / 2}, p_{1 / 2}, s_{1 / 2}, d_{5 / 2}\right)$ space to $\sim 580 \mathrm{keV}$ in the truncated space of $p_{3 / 2}$ waves. Dealing with a broader resonance facilitates benchmarking with CS back-rotation results and helps pin down the dependence on $\mathrm{HO}$ parameters in GSM calculations. The $p_{3 / 2}$ continuum was discretized with a maximum of 60 points. This ensures fully converged results with respect to the Berggren basis (both the number of discretization points and $k_{\max }$ ).

\section{BACK ROTATION: FROM COMPLEX SCALING TO GAMOW STATES}

Even if the energies of resonant states in CS and the GSM are the same, the wave functions are different [see Eqs. (9) and (10)]. This implies that the respective expectation values of an observable $\hat{O}$ in states $\Psi\left(\boldsymbol{r}_{13}, \boldsymbol{r}_{23}\right)$ and $\Psi_{\vartheta}\left(\boldsymbol{r}_{13}, \boldsymbol{r}_{23}\right)$ cannot be compared directly. Moreover, when the wave function $\Psi_{\vartheta}\left(\boldsymbol{r}_{13}, \boldsymbol{r}_{23}\right)$ is used, one has to deal with the transformed operator

$$
\hat{O}_{\vartheta}=U(\vartheta) \hat{O} U(\vartheta)^{-1}
$$

In some cases, it is straightforward to derive the transformed operator. For instance, in the calculation of the root-meansquare radius, the transformed operator is $e^{2 i \vartheta} \boldsymbol{r}_{13}^{2}+e^{2 i \vartheta} \boldsymbol{r}_{23}^{2}$. The transformed recoil operator is given by $-e^{-2 i \vartheta} \frac{\hbar^{2}}{m_{3}} \nabla_{\boldsymbol{r}_{13}} \nabla_{\boldsymbol{r}_{23}}$, and the angular correlation function is the mean value of the operator $\delta\left(\theta_{12}-\theta\right)$, where $\theta_{12}$ is the angle between the vectors $\boldsymbol{r}_{13}$ and $\boldsymbol{r}_{23}$. For the radial density, the situation is not that simple and we shall discuss this point in the following.

In order to retrieve the Gamow wave function of the original Schrödinger equation, it is tempting to carry out a direct backrotation of the CS wave function (11):

$$
e^{-i 3 \vartheta} \sum_{\{l j\}} \sum_{A} C_{A}^{\{l j\}}(\vartheta) \chi_{A}^{\{l j\}}\left(e^{-i \vartheta} r_{13}, e^{-i \vartheta} r_{23}\right) \mathcal{Y}_{\{l j\}}^{J M T T_{z}}\left(\boldsymbol{x}_{13}, \boldsymbol{x}_{23}\right) \text {. }
$$

It turns out, however, that this method is numerically unstable. Even for one particle moving in a potential well, the direct back-rotation leads to unphysical large oscillations in the wave function $[17,18]$. To prevent this, a proper regularization procedure needs to be applied [57,58].

The radial density is defined as the mean value of the operator:

$$
\frac{1}{2}\left[\delta\left(r_{13}-r\right)+\delta\left(r_{23}-r\right)\right] .
$$

By using the CS wave function (32) and the Slater-type radial basis functions (12), the density can be cast into the form

$$
\rho_{\vartheta}(r)=r^{2} \sum_{j} C_{j}(\vartheta) r^{n_{j}} \exp \left(-a_{j} r\right),
$$

where $C_{j}(\vartheta)$ are related to the linear expansion parameters (15), obtained from the diagonalization of the complex-scaled Hamiltonian (6). If we consider the direct back-rotated wave function, the radial density is given by

$$
\rho_{\vartheta}^{\text {back }}(r)=e^{-i \vartheta} \tilde{\rho}_{\vartheta}\left(e^{-i \vartheta} r\right),
$$

where

$$
\tilde{\rho}_{\vartheta}(r)=r^{2} \sum_{j} C_{j}(\vartheta) r^{n_{j}} \exp \left(-a_{j} r\right)
$$

The factor $r^{2}$ comes from the volume element when the Dirac delta function in Eq. (33) is integrated. We shall see that the density calculated in this way leads to extremely inaccurate results. In the following, we briefly show how to calculate the density of the original Gamow state using the CS wave function. Illustrative numerical examples will be presented in Sec. IV B.

We may consider Eq. (36) as a definition of a function defined along the non-negative real axis and $\tilde{\rho}_{\vartheta}\left(e^{-i \vartheta} r\right)$ can be viewed as an attempt to extend (36) into the complex plane. Our approach to the analytic continuation is fairly general. Given an arbitrary analytic function which is known only along the real axis with some error, the objective is to establish its value at some complex argument. To this end, we use the Tikhonov and Fourier transformation methods. In our applications, the function in question is the density $\tilde{\rho}_{\vartheta}(r)$, which can represent neutral or charged particles. Indeed, from the point of view of the analytical continuation, it does not matter whether or not the valence particles are charged, since the related procedure is not related to the actual solution of the many-body problem. Of course, if the complex-scaled density is poorly determined, the Tikhonov and Fourier methods will fail. However, if the density $\tilde{\rho}_{\vartheta}(r)$ is calculated with good accuracy, as we shall demonstrate below, the Tikhonov method is superior to direct back-rotation.

Since the coefficients $C_{i}(\vartheta)$ obtained numerically are not accurate enough, and moreover the Slater expansion is always truncated, the analytical continuation of $\tilde{\rho}_{\vartheta}$ is not a simple task. To find a stable solution, we apply a method based on the theory of Fourier transformations. We first extend $\tilde{\rho}_{\vartheta}(r)$ from $(0, \infty)$ to $(-\infty, \infty)$ by means of the mapping

$$
f_{\vartheta}(x)=\tilde{\rho}_{\vartheta}\left(r_{0} e^{-x}\right) .
$$

The Fourier transform of (37) is

$$
\begin{aligned}
\hat{f}_{\vartheta}(\xi) & =\frac{1}{\sqrt{2 \pi}} \int_{-\infty}^{\infty} e^{-i x \xi} f_{\vartheta}(x) d x \\
& =\frac{1}{\sqrt{2 \pi}} \sum_{j} C_{j}(\vartheta) r_{0}^{n_{j}+2} \frac{\Gamma\left(n_{j}+2+i \xi\right)}{\left(r_{0} a_{j}\right)^{n_{j}+2+i \xi}},
\end{aligned}
$$

where $\xi$ and $x$ are dimensionless variables.

Usually, $\hat{f}_{\vartheta}$ is determined with an error, which results in the appearance of high-frequency oscillations in $f_{\vartheta}$. Now we shall apply Tikhonov smoothing [59] to $f_{\vartheta}(x+i y)$. To this end, we perform the analytical continuation of $f_{\vartheta}(x)$ to the complex plane $x+i y[57]$ :

$$
f_{\vartheta}(x+i y)=\frac{1}{\sqrt{2 \pi}} \int_{-\infty}^{\infty} d \xi e^{i(x+i y) \xi} \hat{f}_{\vartheta}(\xi) .
$$




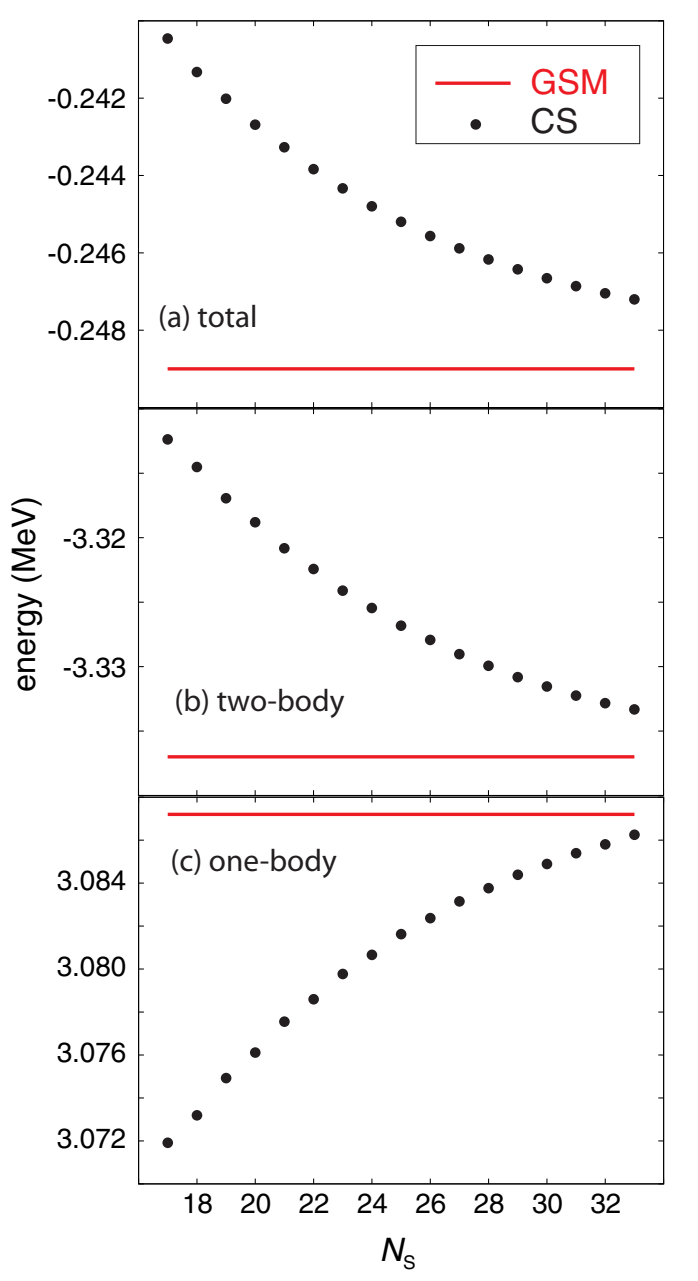

FIG. 3. (Color online) Convergence of the ${ }^{6} \mathrm{He}$ total g.s. energy and two-body and one-body terms, with respect to the number of Slater orbitals, $N_{\mathrm{S}}$, for $\alpha=\beta=0.8$.

Tikhonov regularization [58] removes the ultraviolet noise in Eq. (39) by introducing a smoothing function

$$
f_{\vartheta}^{\mathrm{reg}}(x+i y)=\frac{1}{\sqrt{2 \pi}} \int_{-\infty}^{\infty} e^{i(x+i y) \xi} \frac{\hat{f}_{\vartheta}(\xi)}{1+\kappa e^{-2 y \xi}} d \xi,
$$

where $\kappa$ is the Tikhonov smoothing parameter. In the actual calculation we take $x=-\ln \left(r / r_{0}\right), y=\vartheta$, and $r_{0}=1 \mathrm{fm}$.

\section{RESULTS}

For the neutron-core interaction we employ the KKNN potential [60] and the interaction between the valence neutrons is approximated by the Minnesota force [61]. We study the convergence properties of the CS-Slater method not only for energies of $0_{1}^{+}$and $2_{1}^{+}$of ${ }^{6} \mathrm{He}$ and individual energy components but also for radial properties and spatial correlations.

\section{A. Energies}

According to (4) the total Hamiltonian of ${ }^{6} \mathrm{He}$ is the sum of one-body terms $H_{13}\left(\boldsymbol{r}_{13}\right)+H_{23}\left(\boldsymbol{r}_{23}\right)$ and two-body terms $-\frac{\hbar^{2}}{m_{3}} \nabla_{\boldsymbol{r}_{13}} \nabla_{\boldsymbol{r}_{23}}+V_{12}\left(\boldsymbol{r}_{12}\right)$. Figure 3 illustrates the convergence

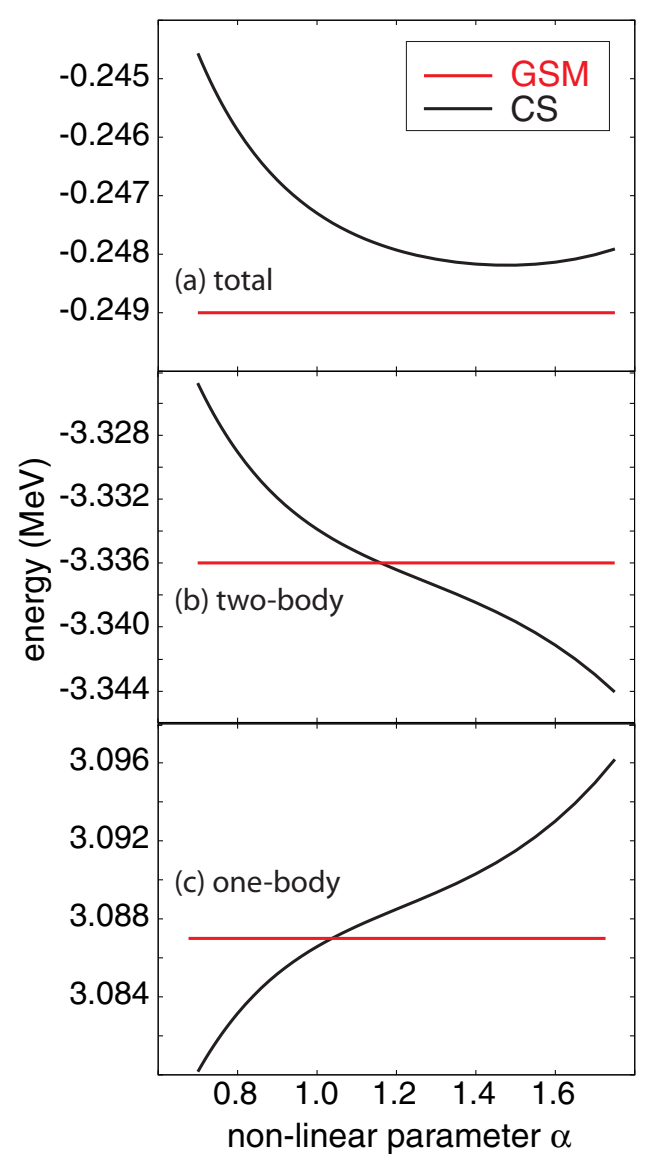

FIG. 4. (Color online) Similar to Fig. 3 but for the nonlinear Slater basis parameter $\alpha=\beta$ for $N_{\mathrm{S}}=27$.

of the CS energies with respect to the basis size $N_{\mathrm{S}} \geqslant n+m$ [see Eq. (12) for notation]. A similar type of restriction was used in Refs. [31,32] in order to avoid the linear dependence of the basis functions. For the nonlinear parameters of the Slater basis we assumed the value $\alpha=\beta=0.8$. The dependence on the Slater basis parameter $\alpha$ is shown in Fig. 4 for $N_{\mathrm{S}}=27$.

In Figs. 3 and 4, horizontal solid lines correspond to GSM results. The maximum difference between CS and GSM energies is of the order of $2 \mathrm{keV}$ for the total energy and two-body and and one-body terms. As can be seen in Fig. 4, two-body and one-body terms have no minima with respect to $\alpha$. This is expected as it is the total energy that is supposed to exhibit a variational minimum, not its individual contributions. The two- and one-body terms coincide with the GSM result for a slightly different variational parameter $(\alpha \sim 1.1)$ than the one that corresponds to the minimum of the total energy $(\alpha=1.5)$. Nevertheless, the difference at the minimum is very small, of the order of $2 \mathrm{keV}$.

Table I displays the energy budget for the bound g.s. configuration of ${ }^{6} \mathrm{He}$ in GSM and CS methods. Even though it is not necessary to use CS for a bound state, we also show values for $\vartheta=0.2$, for reasons that will be explained later in Sec. IV B. In this case, the expectation value of the transformed operator $\hat{O}_{\vartheta}=U(\vartheta) \hat{O} U(\vartheta)^{-1}$ was computed. It is seen that the excellent agreement is obtained between the GSM and 
TABLE I. Energy decomposition of the ${ }^{6} \mathrm{He}$ g.s. Values are in $\mathrm{MeV}$.

\begin{tabular}{lrrc}
\hline \hline$\langle\hat{O}\rangle$ & \multicolumn{1}{c}{ GSM } & CS $(\vartheta=0)$ & CS $(\vartheta=0.2)$ \\
\hline$\langle\hat{H}\rangle$ & -0.249 & -0.247 & $-0.247+i 1.1 \times 10^{-3}$ \\
$\langle\hat{T}\rangle$ & 24.729 & 24.731 & $24.733-i 7.27 \times 10^{-3}$ \\
$\left\langle V_{c-n}\right\rangle$ & -21.642 & -21.645 & $-21.647+i 4.76 \times 10^{-3}$ \\
$\left\langle V_{n n}\right\rangle$ & -2.711 & -2.710 & $-2.710+i 3.11 \times 10^{-3}$ \\
$\left\langle\frac{\vec{p}_{1} \cdot \vec{p}_{2}}{m_{3}}\right\rangle$ & -0.625 & -0.623 & $-0.623+i 5.04 \times 10^{-3}$ \\
\hline \hline
\end{tabular}

both CS variants not only for the total energy but also for all Hamiltonian terms.

We now move on to the $2^{+}$unbound excited state of ${ }^{6} \mathrm{He}$. To assess the accuracy of computing this state in the GSM, we test the sensitivity of calculations to the $\mathrm{HO}$ expansion (30). It is worth noting that in the GSM method only the two-body interaction and recoil terms are treated within the HO expansion. The kinetic term is calculated in the full Berggren basis; hence, the system maintains the correct asymptotic behavior. Moreover, for the $2^{+}$state in the $p_{3 / 2}$ model space, the recoil term vanishes. The resonance position in the CS-Slater method is determined with the $\vartheta$-trajectory method. Figure 5 displays the result of our tests. Overall, we see a weak dependence of the energy and width of the $2^{+}$state predicted in the GSM on the HO expansion parameters $n_{\max }$ and $b$. The increase of $n_{\max }$ from 6 to 28 results in an energy (width) change of $\sim 20 \mathrm{keV}(\sim 10 \mathrm{keV})$. With increasing $n_{\max }$, the results become less dependent on the oscillator length $b$. For the real part of the energy, there appears some stabilization at large values of $n_{\max }$. but the pattern is different for different values of $b$. The most stable results are obtained with $b=2$ $\mathrm{fm}$, where we find a broad plateau for both the energy and the energy modulus $[15,62,63]$ for $n_{\max }>16$. We adopt the value of $b_{\mathrm{opt}}=2 \mathrm{fm}$ for the purpose of further benchmarking.

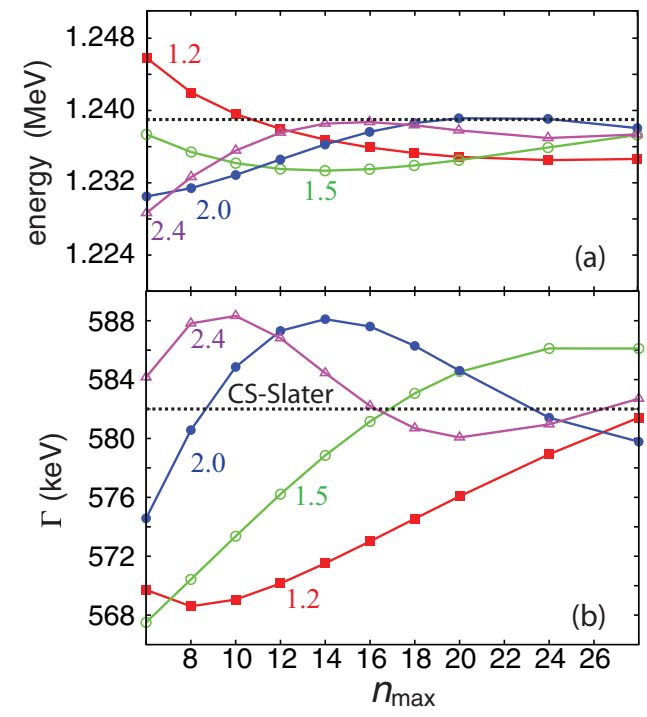

FIG. 5. (Color online) Dependence of the energy (a) and width (b) of the unbound $2_{1}^{+}$state in ${ }^{6} \mathrm{He}$ calculated with the GSM on the HO expansion parameters $n_{\max }$ and $b(=1.2,1.5,2.0$, and $2.4 \mathrm{fm})$ in Eq. (30). The CS-Slater result is marked by a dotted line.
TABLE II. Similar to Table I but for the $2_{1}^{+}$resonance. In GSM calculations, we used $b_{\text {opt }}=2 \mathrm{fm}$ and $n_{\max }=20\left(\mathrm{GSM}_{\mathrm{I}}\right)$ and $n_{\max }=$ $24\left(\mathrm{GSM}_{\mathrm{II}}\right)$. The optimal scaling angle $\vartheta_{\mathrm{opt}}=0.43$ was obtained with the $\vartheta$-trajectory method.

\begin{tabular}{lccc}
\hline \hline$\langle\hat{O}\rangle$ & $\mathrm{CS}\left(\vartheta=\vartheta_{\text {opt }}\right)$ & $\mathrm{GSM}_{\mathrm{I}}$ & $\mathrm{GSM}_{\mathrm{II}}$ \\
\hline$\langle\hat{H}\rangle$ & $1.239-i 0.291$ & $1.239-i 0.292$ & $1.239-i 0.290$ \\
$\langle\hat{T}\rangle$ & $17.340-i 7.949$ & $17.311-i 7.825$ & $17.221-i 7.766$ \\
$\left\langle V_{c-n}\right\rangle$ & $-15.831+i 7.408$ & $-15.805+i 7.288$ & $-15.717+i 7.231$ \\
$\left\langle V_{n n}\right\rangle$ & $-0.270+i 0.250$ & $-0.267+i 0.244$ & $-0.265+i 0.244$ \\
\hline \hline
\end{tabular}

The pattern for the width is similar, with no clear plateau but very small differences at large $n_{\max }$. Such a behavior is not unexpected. While the variational arguments do not apply to the interaction but to the trial wave function $[15,62,63]$, one can demonstrate $[52,54]$ that, while the matrix elements exhibit weak converge with $n_{\max }$, eigenvectors and energies show strong convergence. However, the actual convergence is very slow for broad resonant states.

Based on our tests presented in Fig. 5 we conclude that the numerical error of the GSM method, due to HO expansion, on the energy and width of the $2_{1}^{+}$resonance in ${ }^{6} \mathrm{He}$ is $\sim 2 \mathrm{keV}$. This accuracy is more than needed to carry out the CS-GSM benchmarking.

Table II displays the energy budget for the unbound $2_{1}^{+}$state of ${ }^{6} \mathrm{He}$. We show two variants of GSM calculations in which the interaction was expanded in a $\mathrm{HO}$ basis with $b_{\mathrm{opt}}=2$ fm and $n_{\max }=20\left(\mathrm{GSM}_{\mathrm{I}}\right)$ and $24\left(\mathrm{GSM}_{\mathrm{II}}\right)$. The real parts of the total energy are identical in both methods up to the third digit, and the imaginary parts up to second digit. For the other parts of the Hamiltonian, results are not as precise as for the g.s. calculations in Table I; nevertheless, we obtain an overall satisfactory agreement. It is encouraging, however, that for the total complex energy the agreement is excellent. The benchmarking results presented in this section demonstrate the equivalence of GSM and CS-Slater methods for energies of bound and unbound resonance states. In the following, we shall see that this equivalence also holds for the many-body wave functions.

Our approach can be compared with CS calculations of Ref. [26] in a Gaussian basis in a model space consisting of partial waves up to $d_{5 / 2}$. Their ground-state energy of $-0.244 \mathrm{MeV}$ and $2_{1}^{+}$energy of $1.23-i 0.275 \mathrm{MeV}$ are in a good agreement with our results listed in Tables I and II. This work [26] demonstrated the advantage of using a correlated basis (mixed "T" and "V" Jacobian coordinates) over the single "V"-type basis.

Our g.s. prediction and also those of Ref. [26] do not reproduce the experimental value. The discrepancy occurs (i) because correlations that are of importance for the detailed description of ${ }^{6} \mathrm{He}$ are neglected $[64,65]$ ) and (ii) because of the relatively small number of partial waves employed in our work and Ref. [26]. In the three-body calculation of ${ }^{6} \mathrm{He}$ $[26,64,66]$ - using the same interactions as in our work and performed in a very large model space-the binding energy was found to be -0.73 and $-0.78 \mathrm{MeV}$ with the "V"-type and "V+T"-type coordinates, respectively. In the Lagrange-mesh 


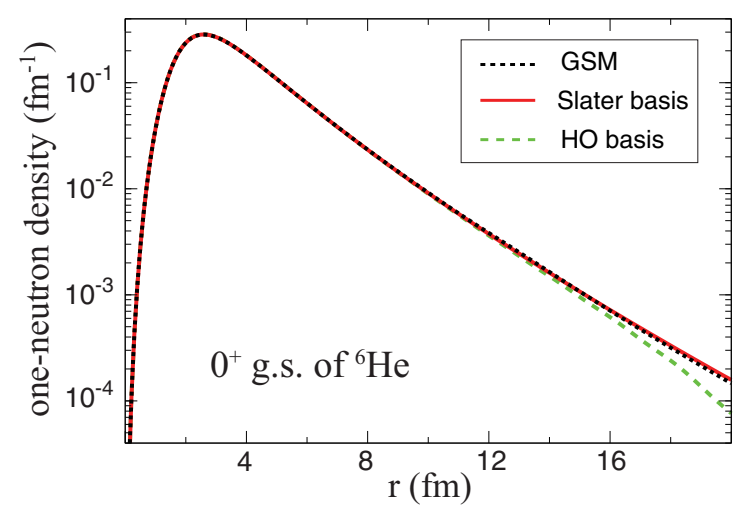

FIG. 6. (Color online) Ground-state one-neutron radial density in ${ }^{6} \mathrm{He}$ predicted with GSM, CS-Slater, and HO basis sets.

calculations using the hyperspherical coordinates [67], the binding energy was further reduced to $-0.87 \mathrm{MeV}$. One may thus say that the hyperspherical description is capable of incorporating more correlations. However, if all calculations are fully converged with respect to both the number of partial waves and the number of radial wave functions, then the discrepancy has to be attributed to some differences in the actual implementation of the model, such as the treatment of the angular-momentum dependence of the $\alpha-n$ potentials [67] or removal of Pauli forbidden states [67,68]. (In this context, it is worth mentioning that a strategy that is often used in nuclear configuration interaction calculations is to choose a specific model space and refit the effective interaction. In this way, some "missing" correlations can be absorbed into the parametrization of the force.) However, since the purpose of our paper is to propose a new numerical technique rather than provide a precise description of the ${ }^{6} \mathrm{He}$ binding mechanism, we have limited ourselves to smaller model spaces to facilitate benchmarking.

\section{B. One-body densities}

To assess the quality of wave functions calculated with the GSM and CS-Slater methods, we first calculate the radial one-neutron density of the g.s. of ${ }^{6} \mathrm{He}$. Figure 6 shows that both methods are consistent with each other and they correctly predict exponential fall-off at large distances. We also display the one-neutron density obtained with the radial part of the wave function (11) spanned by the radial $\mathrm{HO}$ basis states with $b=2 \mathrm{fm}$ and $n_{\max }=18$. As expected, the HO result falls off too quickly at very large distances due to the incorrect asymptotic behavior.

The g.s. of ${ }^{6} \mathrm{He}$ is a bound state; hence, its description does not require a complex rotation of the Hamiltonian. Nevertheless, it is instructive to study the effect of CS on its radial properties. Figure 7 shows the g.s. one-neutron density obtained with the CS-Slater method using $\vartheta=0.1$. For comparison we also display the unscaled $(\vartheta=0)$ density of Fig. 6. We see that the one-particle density is $\vartheta$ dependent and for $\vartheta>0$ it acquires an imaginary part. Since the integral of the density is normalized to 1 , the integral of the imaginary part should be zero. This was checked numerically to be

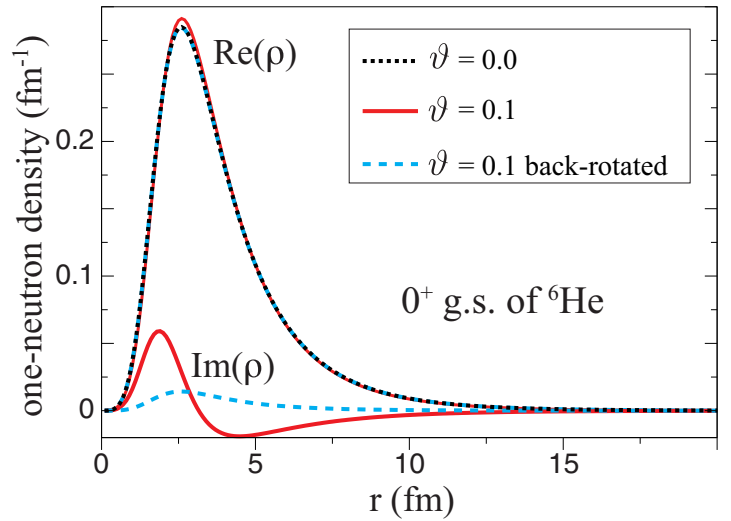

FIG. 7. (Color online) Ground-state one-neutron radial density in ${ }^{6} \mathrm{He}$ predicted with CS-Slater method using $\vartheta=0$ (dotted line) and 0.1 (solid line). The back-rotated $\vartheta=0.1$ result is marked by a dashed line.

indeed the case. Since the back-rotated density should be equivalent to the unscaled or GSM one, its imaginary part should vanish. However, as seen in Fig. 7, the back-rotated density at $\vartheta=0.1$ is nonzero. This is indicative of serious problems with back-rotation in CS, if this method is applied directly $[17,18]$.

In order to investigate back-rotation in more detail, we consider the $2_{1}^{+}$resonance in ${ }^{6} \mathrm{He}$. As in Sec. IV A, we limit ourselves to a $p_{3 / 2}$ model space to better see the effect of backrotation; by adding more partial waves, the $2^{+}$state becomes more localized and the CS density resembles the GSM result. The one-body density derived from the rotated CS solution is very different from the GSM density (see Fig. 8). As the theory implies, the CS density is localized, and the degree of localization increases with $\vartheta$ [17]. To compare with the GSM density, which has outgoing asymptotics, we need to back-rotate the CS radial density.

A comparison of the back-rotated CS-Slater and GSM $2^{+}-$ state densities is presented in Figs. 9 and 10. Here the problem with the back-rotated CS density is far more pronounced than for the g.s. case shown in Fig. 7: at $r>2 \mathrm{fm}$, the real part of

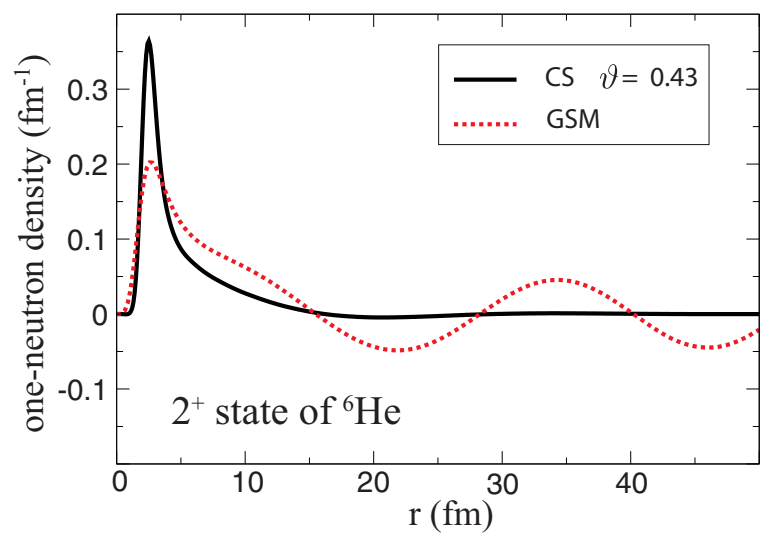

FIG. 8. (Color online) Real part of one-neutron radial density for the unbound $2^{+}$state in ${ }^{6} \mathrm{He}$ obtained in the GSM (solid line) and in the CS-Slater method $\left(\vartheta_{\mathrm{opt}}=0.43\right)$. 


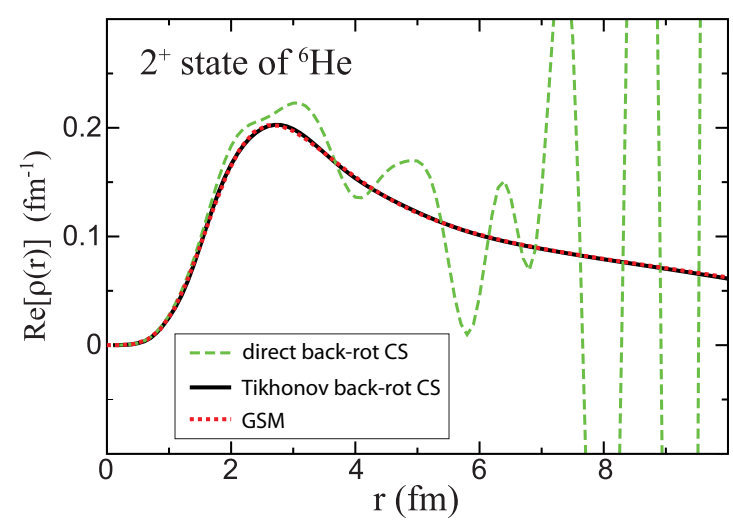

FIG. 9. (Color online) Real part of one-neutron radial density for the unbound $2^{+}$state in ${ }^{6} \mathrm{He}$ obtained in the back-rotated (dashed line) and Tikhonov-regularized-back-rotated (solid) CS-Slater method at $\vartheta_{\text {opt }}$. The GSM density is marked by a dotted line.

the back-rotated density exhibits unphysical oscillations. The magnitude of those oscillations grows with $\vartheta$, even if the basis size is increased. The situation is even worse for the imaginary part of the density, which does not resemble the GSM density at $r>1 \mathrm{fm}$.

The numerical instability of the back-rotated CS wave functions is an example of an ill-posed inverse problem [69]. The amplitudes of the wave function (36) are determined numerically, and the associated errors are amplified during the back-rotation (35), causing instabilities seen in Figs. 9 and 10. Consequently, one needs a regularization method to minimize the errors that propagate from the coefficients to the solution. In this paper, we apply the Fourier analytical continuation and Tikhonov regularization procedure [58,59] described in Sec. III.

We first investigate the Fourier transform (39), which provides us with an analytical continuation of the density. It is understood that if one performs the integral in the full interval $(-\infty, \infty)$, the analytically continued density would also exhibit unwanted oscillations. Indeed, at large negative values of $\xi$ in (39), the exponent may become very large, amplifying numerical errors of the Fourier transform $\hat{f}_{\vartheta}(\xi)$ and causing numerical instabilities. For this reason we cut the

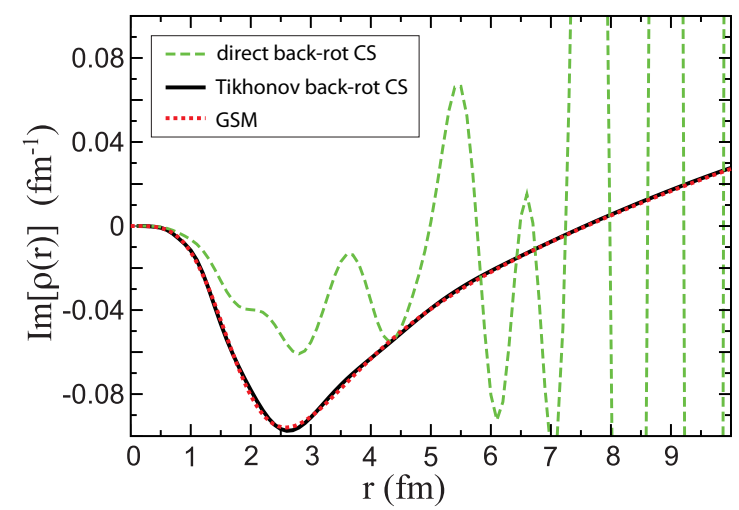

FIG. 10. (Color online) Similar to Fig. 9 but for the imaginary part of the density.

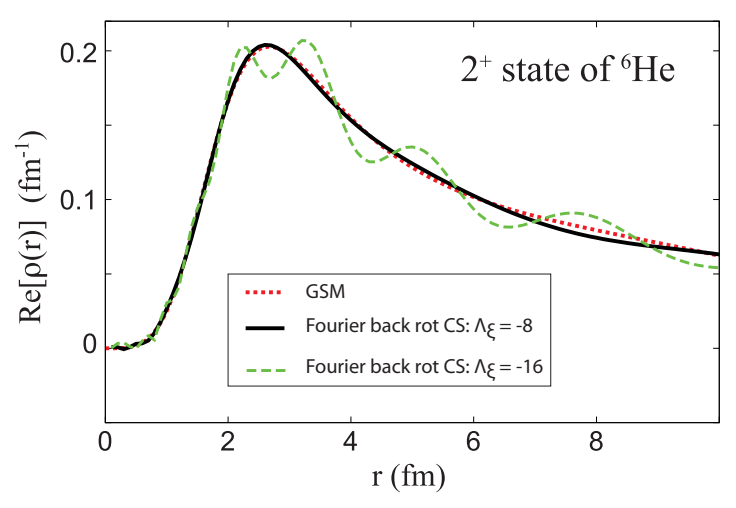

FIG. 11. (Color online) Real part of one-neutron radial density for the $2^{+}$resonance in ${ }^{6} \mathrm{He}$ obtained in the back-rotated CS-Slater method using the Fourier-regularized analytical continuation with $\Lambda_{\xi}=-8$ (solid line) and $\Lambda_{\xi}=-16$ (dashed line). The GSM density is marked by a dotted line.

lower range of $\xi$ in Eq. (39) to obtain the expression for the Fourier-regularized function:

$$
f_{\vartheta}(x+i y)=\frac{1}{\sqrt{2 \pi}} \int_{\Lambda_{\xi}}^{\infty} e^{i(x+i y) \xi} \hat{f}_{\vartheta}(\xi) d \xi
$$

Figure 11 compares the GSM density of the $2^{+}$resonance in ${ }^{6} \mathrm{He}$ with back-rotated CS-Slater densities obtained using the Fourier-regularized analytical continuation. By taking the cutoff parameter $\Lambda_{\xi}=-8$ we obtain a density that is almost identical to that of the GSM. With $\Lambda_{\xi}=-16$, the analytically continued density starts to oscillate around the GSM result, and with even larger negative values of cutoff used, the highfrequency components become amplified and eventually one recoups the highly fluctuating direct back-rotation result of Fig. 9.

In the Tikhonov method, the sharp cutoff $\Lambda_{\xi}$ is replaced by a smooth cutoff (or filter) characterized by a smoothing parameter $\kappa$. In Eq. (40) this has been achieved by means of the damping function (regulator) $[1+\kappa \exp (-2 y \xi)]^{-1}$ that attenuates large negative values of $\xi$, with the parameter $\kappa$ controlling the degree of regularization. The functional form of the regulator is not unique; it depends on the nature of the problem. In the applications presented in this study, the analytically continued density coincides with the $\vartheta$ independent result for $\kappa=4 \times 10^{-4}$, which also corresponds to the original resonant GSM solution. The results presented in Figs. 9 and 10 demonstrate that both real and imaginary parts of the resonance's density obtained in the Tikhonov-regularized CS-Slater method are in excellent agreement with the GSM result.

To understand in more detail the mechanism behind the Tikhonov regularization, in Fig. 12 we display the real part of the integrand in Eq. (39) at $r=20 \mathrm{fm}, \vartheta_{\text {opt }}=0.43$, and $\kappa=0$ (no regularization) and $\kappa=4 \times 10^{-7}$ and $4 \times 10^{-4}$. In the absence of a regulator, at $\xi<-10$ the integrand exhibits oscillations with increasing amplitude. Below $\xi=-8$, all three variants of calculations are very close; this explains the excellent agreement between GSM and back-rotated CS results in Fig. 11 with $\Lambda_{\xi}=-8$. In short, with the Tikhonov 


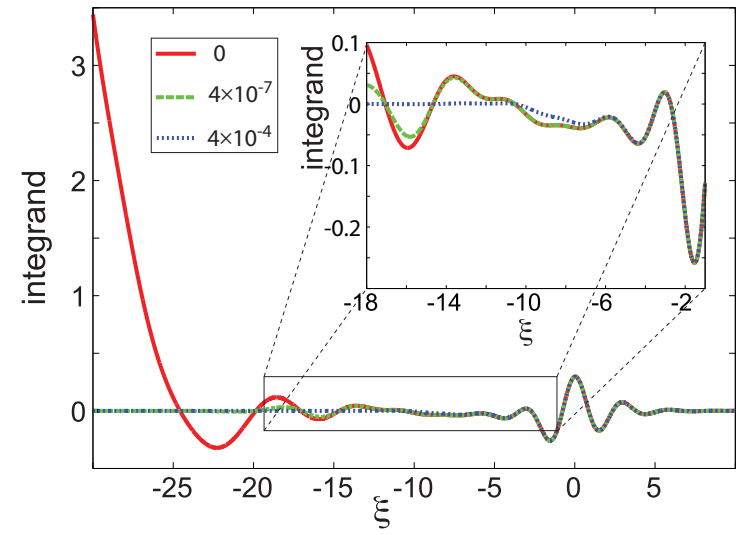

FIG. 12. (Color online) Real part of the integrand in Eq. (40), calculated at $r=20 \mathrm{fm}, \vartheta_{\text {opt }}=0.43$, and $\kappa=0,4 \times 10^{-7}$, and $4 \times$ $10^{-4}$. To see the detailed behavior at small negative values of $\xi$, the region of $-18 \leqslant \xi \leqslant-1$ is shown in the inset.

method, large values of the integrand at large negative values of $\xi$ are suppressed, thus enabling us to obtain an excellent reproduction of the resonant density in the GSM.

It is instructive to study the behavior of the analytically continued back-rotated CS density for different Tikhonov regularization parameters $\kappa$. As mentioned earlier, the value $\kappa=4 \times 10^{-4}$ was found to be optimal; i.e., it produces the CS-Slater densities that are closest to those of GSM. As seen in Fig. 13, for smaller values of $\kappa$, the damping function is too small to eliminate the oscillations at large negative $\xi$ values. This is also depicted in Fig. 12, where for $\kappa=4 \times 10^{-7}$ unwanted oscillations of the integrand appear around $\xi \sim 16$. For larger values of $\kappa$, the integral is over-regulated and produces a suppressed density profile. Similar patterns of $\kappa$ dependence have been found in other studies [70-73].

The behavior seen in Fig. 13 suggests a way to determine the optimal value of the smoothing parameter $\kappa$, regardless of the availability of the GSM result. The idea behind our method is presented in Fig. 14, which shows the values of $\rho(r)$ at two chosen large distances $r_{\kappa}$ (here $r_{\kappa}=3$ and $6 \mathrm{fm}$ ) versus $\kappa$ in a fairly broad range. As expected, at large and small values of

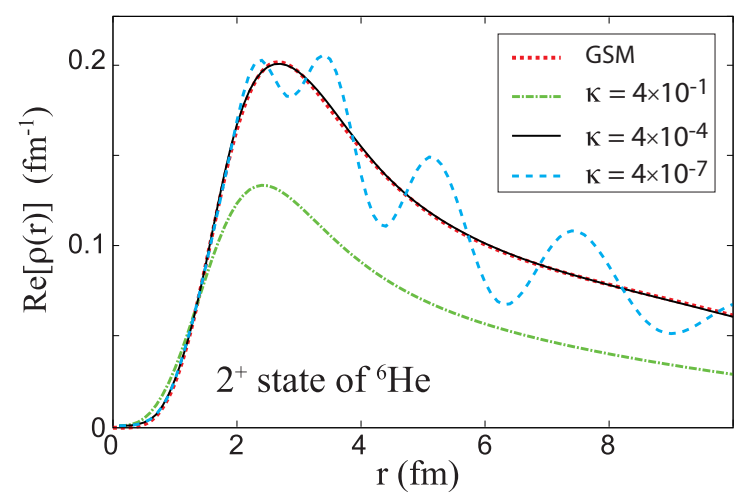

FIG. 13. (Color online) Real part of one-neutron radial density for the $2^{+}$resonance in ${ }^{6} \mathrm{He}$ obtained in the back-rotated CS-Slater method using the Tikhonov regularization with several values of smoothing parameter $\kappa$.

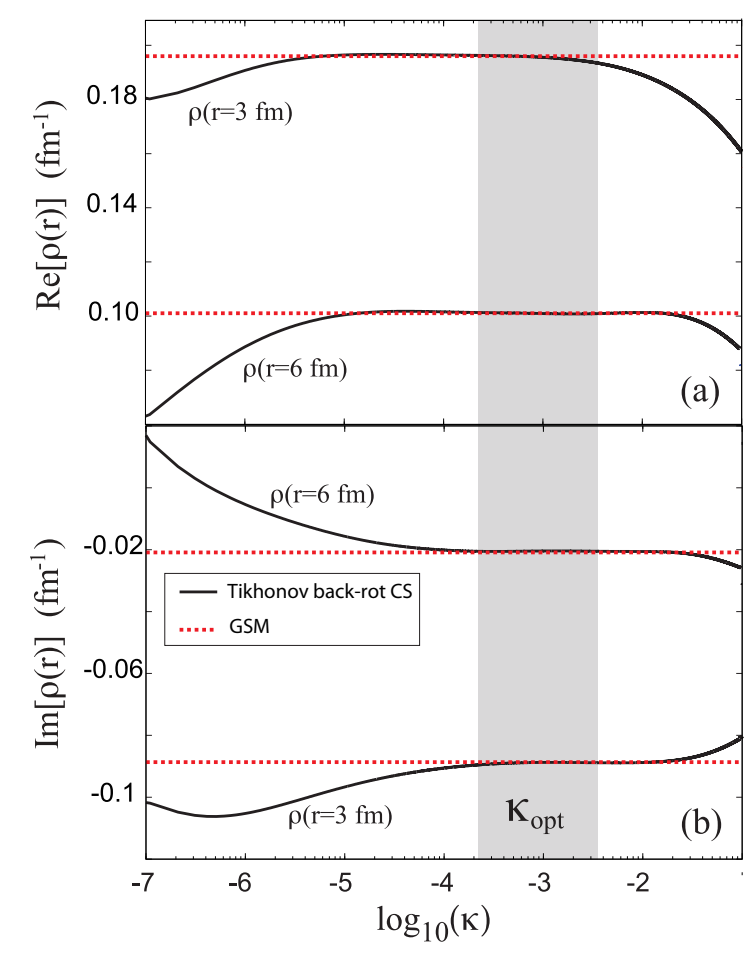

FIG. 14. (Color online) Real (a) and imaginary (b) parts of oneneutron radial density at $r=3$ and $6 \mathrm{fm}$ for the $2^{+}$resonance in ${ }^{6} \mathrm{He}$, as a function of the Tikhonov regularization parameter $\kappa$. In an intermediate region of $\kappa$ values (gray shading), plateaus appear that coincide with the GSM results.

$\kappa, \rho\left(r_{\kappa}\right)$ shows strong variations with the Tikhonov smoothing parameter. However, at intermediate values, a plateau in $\rho\left(r_{\kappa}\right)$ appears that nicely coincides with the GSM results. Our optimal choice, $\kappa_{\mathrm{opt}}=4 \times 10^{-4}$, belongs to this plateau.

\section{Two-body angular densities}

The two-body density contains information about twoneutron correlations. It is defined as [74]

$$
\rho\left(\boldsymbol{r}, \boldsymbol{r}^{\prime}\right)=\left\langle\Psi\left|\delta\left(\boldsymbol{r}-\boldsymbol{r}_{1}\right) \delta\left(\boldsymbol{r}^{\prime}-\boldsymbol{r}_{2}\right)\right| \Psi\right\rangle .
$$

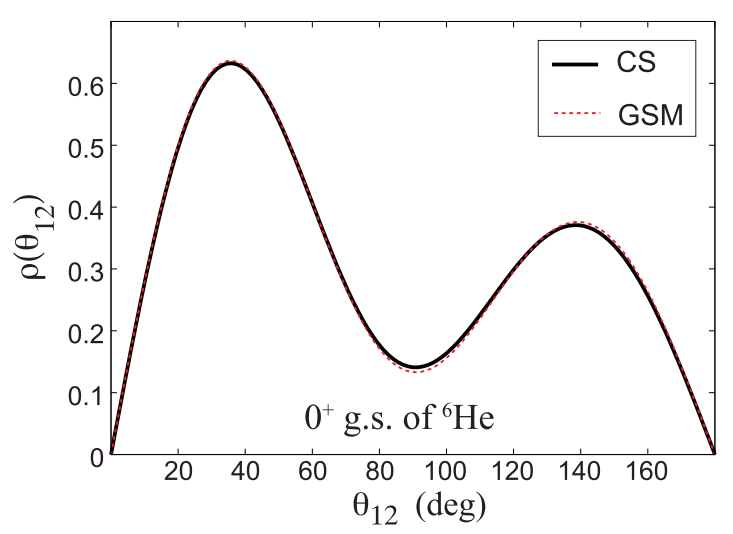

FIG. 15. (Color online) Angular two-neutron density for the ${ }^{6} \mathrm{He}$ g.s. predicted in the GSM and CS-Slater methods. 


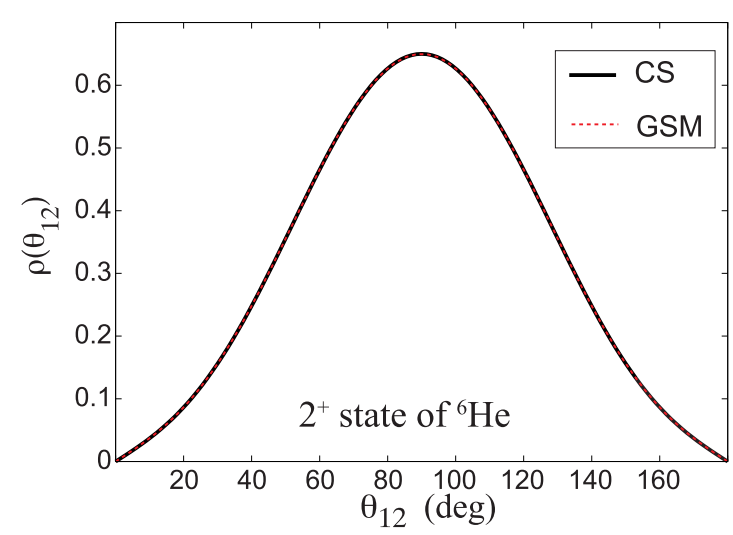

FIG. 16. (Color online) Similar as in Fig. 15 but for the $2^{+}$ resonance.

In spherical coordinates, it is convenient to introduce [56]

$$
\rho\left(r, r^{\prime}, \theta\right)=\left\langle\Psi\left|\delta\left(r_{1}-r\right) \delta\left(r_{2}-r^{\prime}\right) \delta\left(\theta_{12}-\theta\right)\right| \Psi\right\rangle,
$$

with $r_{1}\left(r_{2}\right)$ being the distance between the core and the first (second) neutron and $\theta_{12}$ being the opening angle between the two neutrons. The density $\rho\left(r, r^{\prime}, \theta\right)$ differs from the two-particle density (42) by the absence of the Jacobian $8 \pi^{2} r^{2} r^{\prime 2} \sin \theta$. Consequently, the two-body density is normalized according to

$$
\int \rho\left(r, r^{\prime}, \theta\right) d r d r^{\prime} d \theta=1
$$

In practical applications, (43) is calculated and plotted for $r=r^{\prime}$.

By parametrizing the wave function in terms of the distance $r$ from the core nucleus and the angle $\theta$ between the valence particles, one is able to investigate the particle correlations in the halo nucleus. Such calculations were performed recently [56] to explain the observed charge radii differences in helium halo nuclei [75]. To study angular correlations between valence particles, we introduce the angular density

$$
\rho\left(\theta_{12}\right)=\int d r \int d r^{\prime} \rho\left(r, r^{\prime}, \theta_{12}\right) .
$$

Figures 15 and 16 display $\rho\left(\theta_{12}\right)$ for the g.s. and $2_{1}^{+}$resonance, respectively. The agreement between GSM and CS-Slater methods is excellent. It is worth noting that the calculation of the angular density in the CS-Slater framework does not require back-rotation. Indeed, since the CS operator (5) acts only on the radial coordinates, once they are integrated out one obtains the unscaled result.

\section{CONCLUSIONS}

In this work, we introduce a new efficient CS method in a Slater basis to treat open many-body systems. We apply the technique to the two-neutron halo nucleus ${ }^{6} \mathrm{He}$ considered as a three-body problem. The interaction between valence neutrons is modeled by a finite-range Minnesota force.
To benchmark the new method, we computed the weakly bound g.s. and $2_{1}^{+}$resonance in ${ }^{6} \mathrm{He}$ in both CS-Slater and GSM methods. We carefully studied the numerical accuracy of both methods and found it more than sufficient for the purpose of benchmarking. Based on our tests, we find both approaches to be equivalent for all the quantities studied. In a parallel development [76,77], the CS method in a Gaussian basis [78] has been compared with the GSM method for ${ }^{6} \mathrm{He}$ and ${ }^{6} \mathrm{Be}$ and a good overall agreement has been found.

An important aspect of our study was the application of the Tikhonov regularization technique to CS-Slater back-rotated wave functions in order to minimize the ultraviolet numerical noise at finite scaling angles $\vartheta$. We traced back the origin of high-frequency oscillations to the high-frequency part of the Fourier transform associated with the analytic continuation of the CS wave function and found a practical way to determine the smoothing parameter defining the Tikhonov regularization. The applied stabilization method allows us to reconstruct the correct radial asymptotic behavior by using localized complex-scaled wave functions. This can be of importance when calculating observables that are directly related to the asymptotic behavior of the system, such as cross sections or decay widths. The proposed method is valid not only for narrow resonances (as for example Ref. [19]) but also for broad resonant states, such as the excited $2^{+}$state of ${ }^{6} \mathrm{He}$.

In the near future, we intend to include the internucleon distance $r_{12}$ in Eq. (12) to obtain the full Hylleraas basis that promises somehow improved numerical convergence and higher accuracy. This will enable us to formulate a reaction theory directly in Hylleraas coordinates. The near-term application could include $\alpha+d$ elastic scattering and radiative capture reactions as in Ref. [28] and atomic applications such as electron-hydrogen scattering.

\section{ACKNOWLEDGMENTS}

Useful discussions with G. W. F Drake and M. Płoszajczak are gratefully acknowledged. This work was supported by the US Department of Energy under Contract No. DEFG02-96ER40963 and by the TÁMOP-4.2.2.C-11/1/KONV2012-0001 project. The project has been supported by the European Union, co-financed by the European Social Fund. An allocation of advanced computing resources was provided by the National Science Foundation. Computational resources were provided by the National Center for Computational Sciences (NCCS) and the National Institute for Computational Sciences (NICS).

\section{APPENDIX: RADIAL INTEGRALS}

To simplify the radial integral (21) we use the explicit form of the Legendre polynomial $P_{\lambda}(x)=\sum_{n=0}^{\lambda} \eta_{\lambda, n} x^{n}$ and 
the binomial theorem to get

$$
\begin{aligned}
I^{(\lambda)}\left(n_{13}, n_{23}\right)= & \sum_{n=0}^{\lambda} \eta_{\lambda, n} 2^{-n} \sum_{k=0}^{n}\left(\begin{array}{l}
n \\
k
\end{array}\right) \sum_{m=0}^{k}(-1)^{k}\left(\begin{array}{c}
k \\
m
\end{array}\right) \int_{0}^{\infty} d r_{13} r_{13}^{n_{13}+n-2 k+1} e^{-a_{13} r_{13}} \\
& \times \int_{0}^{\infty} d r_{23} r_{23}^{n_{23}+2 k-2 m-n+1} e^{-a_{23} r_{23}} \int_{\left|r_{13}-r_{23}\right|}^{r_{13}+r_{23}} d r_{12} r_{12}^{2 m+1} V_{12}\left(r_{12}\right) .
\end{aligned}
$$

Now we make a variable transformation from the relative coordinates $r_{12}, r_{13}$, and $r_{23}$ to the Hylleraas coordinates $s, t$, and $u$ defined by the equations $s=r_{13}+r_{23}, t=r_{13}-r_{23}$, and $u=r_{12}$. Expressed in $s, t$, and $u$, the radial volume element becomes $d \tau_{r}=\frac{1}{8}\left(s^{2}-t^{2}\right) d s d u d t$, and (A1) can be written as

$$
\begin{aligned}
I^{(\lambda)}\left(n_{13}, n_{23}\right)= & \sum_{n=0}^{\lambda} \eta_{\lambda, n} 2^{-n} \sum_{k=0}^{n}\left(\begin{array}{l}
n \\
k
\end{array}\right) \sum_{m=0}^{k}(-1)^{k}\left(\begin{array}{l}
k \\
m
\end{array}\right) \sum_{k_{13}=0}^{N_{13}+1} \sum_{k_{23}=0}^{N_{23}+1} 2^{-3-N_{13}-N_{23}}\left(\begin{array}{c}
N_{13}+1 \\
k_{13}
\end{array}\right)\left(\begin{array}{c}
N_{23}+1 \\
k_{23}
\end{array}\right)(-1)^{k_{23}} \\
& \times \int_{0}^{\infty} d s e^{-a s} s^{N_{13}+N_{23}+2-k_{13}-k_{23}} \int_{0}^{s} d u u^{N_{12}} V_{12}(u) \int_{0}^{u} d t t^{k_{13}+k_{23}} e^{-b t},
\end{aligned}
$$

where $a=\frac{1}{2}\left(a_{13}+a_{23}\right), b=\frac{1}{2}\left(a_{13}-a_{23}\right), N_{12}=2 m+1, N_{13}=n_{13}+n-2 k$, and $N_{23}=n_{23}+2 k-2 m-n$. With the help of the integral

$$
I\left(n_{s}, n_{t}, n_{u}, a, b\right)=\int_{0}^{\infty} d s s^{n_{s}} e^{-a s} \int_{0}^{s} d u u^{n_{u}} V_{12}(u) \int_{0}^{u} d t t^{n_{t}} e^{-b t}
$$

we can write

$$
\begin{aligned}
I^{(\lambda)}\left(n_{13}, n_{23}\right)= & \sum_{n=0}^{\lambda} \eta_{\lambda, n} 2^{-n} \sum_{k=0}^{n}\left(\begin{array}{l}
n \\
k
\end{array}\right) \sum_{m=0}^{k}(-1)^{k}\left(\begin{array}{l}
k \\
m
\end{array}\right) \sum_{k_{13}=0}^{N_{13}+1} \sum_{k_{23}=0}^{N_{23}+1}\left(\begin{array}{c}
N_{13}+1 \\
k_{13}
\end{array}\right)\left(\begin{array}{c}
N_{23}+1 \\
k_{23}
\end{array}\right)(-1)^{k_{23}} 2^{-3-N_{13}-N_{23}} \\
& \times I\left(N_{13}+N_{23}+2-k_{13}-k_{23}, k_{13}+k_{23}, N_{12}, a, b\right) .
\end{aligned}
$$

As the integral over $t$ in Eq. (A3) can be carried out analytically and the integral over $u$ can be computed by using

$$
\frac{d}{d s}\left(-\frac{1}{a^{n_{s}+1}} \Gamma\left(n_{s}+1, a s\right)\right)=e^{-a s} s^{n_{s}}
$$

one gets

$$
I\left(n_{s}, n_{t}, n_{u}, a, b\right)=\frac{1}{\left(n_{t}+1\right) a^{n_{s}+1}} \int_{0}^{\infty} d s \Gamma\left(n_{s}+1, a s\right) s^{n_{u}+n_{t}+1} V_{12}(s) \mathrm{M}\left(n_{t}+1, n_{t}+2,-b s\right),
$$

where $\mathrm{M}\left(n_{t}+1, n_{t}+2,-b s\right)$ is the regular confluent hypergeometric function and $\Gamma\left(n_{s}+1, a s\right)$ is the incomplete Gamma function [79]. Expressing these two special functions as finite sums of elementary functions one finally arrives at the compact general expression

$$
I\left(n_{s}, n_{t}, n_{u}, a, b\right)=\frac{n_{s} ! n_{t} !}{a^{n_{s}+1} b^{n_{t}+1}} \sum_{k=0}^{n_{s}} \frac{a^{k}}{k !} \int_{0}^{\infty} d s e^{-a s} s^{n_{u}+k} V(s)\left[1-e^{-b s} \sum_{n=0}^{n_{t}} \frac{(b s)^{n}}{n !}\right],
$$

which is valid for any form factor $V(r)$. It is immediately seen that for $b=0$ (A7) simplifies to

$$
I\left(n_{s}, n_{t}, n_{u}, a, 0\right)=\frac{n_{s} !}{a^{n_{s}+1}\left(n_{t}+1\right)} \sum_{k=0}^{n_{s}} \frac{a^{k}}{k !} \int_{0}^{\infty} d s e^{-a s} V(s) s^{n_{u}+n_{t}+k+1} .
$$

To compute (A7) with a Gaussian form factor $V(s)=\exp \left(-f s^{2}\right)$, we use

$$
\int_{0}^{\infty} d s \exp \left(-a s-f s^{2}\right) s^{n}=(-1)^{n} K^{(n)}(a)
$$

where $K^{(n)}(z)=\frac{d^{n}}{d z^{n}} K(z)$ with

$$
K(z)=\frac{1}{2} \sqrt{\frac{\pi}{f}} \exp \left(\frac{z^{2}}{4 f}\right) \operatorname{Erfc}\left(\frac{z}{2 \sqrt{f}}\right) .
$$


Expressed in terms of the parabolic cylinder function $D_{-n-1}(z)[80], K^{(n)}(z)$ is

$$
K^{(n)}(z)=\frac{1}{2} \sqrt{\frac{\pi}{f}}\left(\frac{1}{2 \sqrt{f}}\right)^{n} \frac{(-1)^{n} n ! 2^{(n+1) / 2}}{\sqrt{\pi}} \exp \left[z^{2} /(8 f)\right] D_{-n-1}(z / \sqrt{2 f}) .
$$

[1] N. Michel, W. Nazarewicz, J. Okołowicz, and M. Płoszajczak, J. Phys. G 37, 064042 (2010).

[2] A. T. Kruppa and W. Nazarewicz, Phys. Rev. C 69, 054311 (2004).

[3] I. J. Thompson and F. Nunes, Nuclear Reactions for Astrophysics Principles, Calculation and Applications of Low-Energy Reactions (Cambridge University Press, Cambridge, England, 2009).

[4] A. Deltuva, A. C. Fonseca, and S. K. Bogner, Phys. Rev. C 77, 024002 (2008).

[5] S. Baroni, P. Navrátil, and S. Quaglioni, Phys. Rev. C 87, 034326 (2013); S. Quaglioni and P. Navrátil, Phys. Rev. Lett. 101, 092501 (2008); Phys. Rev. C 79, 044606 (2009).

[6] K. M. Nollett, S. C. Pieper, R. B. Wiringa, J. Carlson, and G. M. Hale, Phys. Rev. Lett. 99, 022502 (2007).

[7] G. Hagen and N. Michel, Phys. Rev. C 86, 021602 (2012).

[8] G. Papadimitriou, J. Rotureau, N. Michel, M. Płoszajczak, and B. R. Barrett, Phys. Rev. C 88, 044318 (2013).

[9] G. Hagen, M. Hjorth-Jensen, G. R. Jansen, R. Machleidt, and T. Papenbrock, Phys. Rev. Lett. 108, 242501 (2012); 109, 032502 (2012).

[10] N. Michel, W. Nazarewicz, M. Płoszajczak, and T. Vertse, J. Phys. G: Nucl. Part. Phys. 36, 013101 (2009).

[11] H. Feshbach, Ann. Phys. (NY) 19, 287 (1962).

[12] J. Okołowicz, M. Płoszajczak, and I. Rotter, Phys. Rep. 374, 271 (2003).

[13] A. Volya and V. Zelevinsky, Phys. Rev. C 74, 064314 (2006).

[14] Y. Ho, Phys. Rep. 99, 1 (1983).

[15] N. Moiseyev, Phys. Rep. 302, 212 (1998).

[16] S. Aoyama, T. Myo, K. Katō, and K. Ikeda, Prog. Theor. Phys. 116, 1 (2006).

[17] R. Lefebvre, J. Mol. Struct.: THEOCHEM 166, 17 (1988).

[18] A. Csótó, B. Gyarmati, A. T. Kruppa, K. F. Pál, and N. Moiseyev, Phys. Rev. A 41, 3469 (1990).

[19] R. Lefebvre, Phys. Rev. A 46, 6071 (1992).

[20] A. Buchleitner, B. Grémaud, and D. Delande, J. Phys. B 27, 2663 (1994).

[21] J. N. Bardsley, Int. J. Quantum Chem. 14, 343 (1978).

[22] N. Moiseyev and C. Corcoran, Phys. Rev. A 20, 814 (1979).

[23] K. Varga, J. Mitroy, J. Z. Mezei, and A. T. Kruppa, Phys. Rev. A 77, 044502 (2008).

[24] A. T. Kruppa, R. G. Lovas, and B. Gyarmati, Phys. Rev. C 37, 383 (1988).

[25] A. T. Kruppa and K. Katō, Prog. Theor. Phys. 84, 1145 (1990).

[26] A. Aoyama, S. Mukai, K. Katō, and K. Ikeda, Prog. Theor. Phys 93, 99 (1995); 94, 343 (1995).

[27] T. Myo, K. Katō, S. Aoyama, and K. Ikeda, Phys. Rev. C 63, 054313 (2001).

[28] Y. Kikuchi, N. Kurihara, A. Wano, K. Katō, T. Myo, and M. Takashina, Phys. Rev. C 84, 064610 (2011).

[29] E. A. Hylleraas, Z. Phys. 54, 347 (1929).

[30] M. B. Ruiz, Int. J. Quantum Chem. 101, 246 (2004).

[31] G. Drake, Phys. Scr. T 83, 83 (1999).
[32] G. W. F. Drake, M. M. Cassar, and R. A. Nistor, Phys. Rev. A 65, 054501 (2002).

[33] V. I. Korobov, Phys. Rev. A 61, 064503 (2000).

[34] J. C. Slater, Phys. Rev. 36, 57 (1930).

[35] M. A. Caprio, P. Maris, and J. P. Vary, Phys. Rev. C 86, 034312 (2012).

[36] F. Nunes, J. Christley, I. Thompson, R. Johnson, and V. Efros, Nucl. Phys. A 609, 43 (1996).

[37] J. Aguilar and J. M. Combes, Commun. Math. Phys. 22, 266 (1971).

[38] E. Balslev and J. M. Combes, Commun. Math. Phys. 22, 280 (1971).

[39] R. Lazauskas and J. Carbonell, Phys. Rev. C 84, 034002 (2011).

[40] R. Lazauskas, Phys. Rev. C 86, 044002 (2012).

[41] K. B. Bravaya, D. Zuev, E. Epifanovsky, and A. I. Krylov, J. Chem. Phys. 138, 124106 (2013).

[42] W. P. Reinhardt, Annu. Rev. Phys. Chem 33, 223 (1982).

[43] R. D. Lawson, Theory of the Nuclear Shell Model (Clarendon, Oxford, 1980).

[44] V. D. Éfros, Yad. Fiz. 17, 988 (1973) [Sov. J. Nucl. Phys. 17, 517 (1973)].

[45] V. D. Éfros, Zh. Eksp. Teor. Fiz. 90, 10 (1986) [JETP 63, 5 (1986)].

[46] G. W. F. Drake, Phys. Rev. A 18, 820 (1978).

[47] A. M. Frolov and V. H. Smith, Phys. Rev. A 53, 3853 (1996).

[48] T. Berggren, Nucl. Phys. A 109, 265 (1968).

[49] P. Lind, Phys. Rev. C 47, 1903 (1993); T. Berggren and P. Lind, ibid. 47, 768 (1993).

[50] B. Gyarmati and T. Vertse, Nucl. Phys. A 160, 523 (1971).

[51] M. Moshinsky, Nucl. Phys. 13, 104 (1959); T. Brody, G. Jacob, and M. Moshinsky, ibid. 17, 16 (1960); G. Kamuntavičius, R. Kalinauskas, B. Barrett, S. Mickevičius, and D. Germanas, Nucl. Phys. A 695, 191 (2001).

[52] G. Hagen, M. Hjorth-Jensen, and N. Michel, Phys. Rev. C 73, 064307 (2006).

[53] B. Gyarmati and A. T. Kruppa, Phys. Rev. C 34, 95 (1986).

[54] N. Michel, W. Nazarewicz, and M. Płoszajczak, Phys. Rev. C 82, 044315 (2010).

[55] S. Saito, Prog. Theor. Phys. 41, 705 (1969).

[56] G. Papadimitriou, A. T. Kruppa, N. Michel, W. Nazarewicz, M. Płoszajczak, and J. Rotureau, Phys. Rev. C 84, 051304 (2011).

[57] C.-L. Fu, F.-F. Dou, X.-L. Feng, and Z. Qian, Inverse Problems 24, 065003 (2008).

[58] C.-L. Fu, Z.-L. Deng, X.-L. Feng, and F.-F. Dou, SIAM J. Numer. Anal. 47, 2982 (2009).

[59] A. N. Tikhonov, Sov. Math. Dokl. 4, 1035 (1963).

[60] K. Kanada, T. Kaneko, S. Nagata, and M. Nomoto, Prog. Theor. Phys. 61, 1327 (1979).

[61] D. Thompson, M. Lemere, and Y. Tang, Nucl. Phys. A 286, 53 (1977).

[62] N. Moiseyev, P. Certain, and F. Weinhold, Mol. Phys. 36, 1613 (1978). 
[63] J. Rotureau, N. Michel, W. Nazarewicz, M. Płoszajczak, and J. Dukelsky, Phys. Rev. C 79, 014304 (2009).

[64] D. Baye, Nucl. Phys. A 627, 305 (1997).

[65] A. Csótó, Phys. Rev. C 48, 165 (1993).

[66] D. Baye, M. Kruglanski, and M. Vincke, Nucl. Phys. A 573, 431 (1994).

[67] P. Descouvemont, C. Daniel, and D. Baye, Phys. Rev. C 67, 044309 (2003).

[68] I. J. Thompson, B. V. Danilin, V. D. Efros, J. S. Vaagen, J. M. Bang, and M. V. Zhukov, Phys. Rev. C 61, 024318 (2000).

[69] A. Tikhonov and A. Goncharsky, Ill-posed Problems in the Natural Sciences (Oxford University Press, Oxford, 1987).

[70] P. C. Hansen, SIAM Rev. 34, 561 (1992).

[71] D. P. O'Leary, SIAM J. Sci. Comput. 23, 1161 (2001).

[72] G. H. Golub, M. Heath, and G. Wahba, Technometrics 21, 215 (1979).
[73] H. G. Golub, P. C. Hansen, and D. P. O'Leary, SIAM J. Matrix Anal. Appl. 21, 185 (1999).

[74] R. D. Viollier and J. D. Walecka, Acta Phys. Pol. B 8, 25 (1977); R. Schiavilla, D. Lewart, V. Pandharipande, S. C. Pieper, R. Wiringa, and S. Fantoni, Nucl. Phys. A 473, 267 (1987); G. Bertsch and H. Esbensen, Ann. Phys. (NY) 209, 327 (1991).

[75] Z.-T. Lu, P. Mueller, G. W. F. Drake, W. Nörtershäuser, S. C. Pieper, and Z.-C. Yan, Rev. Mod. Phys. 85, 1383 (2013).

[76] H. Masui, K. Katō, and K. Ikeda, J. Phys.: Conf. Ser. 111, 012029 (2008).

[77] H. Masui, K. Katō, N. Michel, and M. Płoszajczak (personal communication).

[78] E. Hiyama, Y. Kino, and M. Kamimura, Prog. Part. Nucl. Phys. 51, 223 (2003).

[79] M. Abramowitz and I. Stegun, Handbook of Mathematical Functions (Dover, New York, 1965).

[80] J. Segura and A. Gil, Comput. Phys. Commun. 115, 69 (1998). 